\title{
Light Higgsino Dark Matter in the MSSM on D-branes
}

\author{
Van E. Mayes ${ }^{1}$ and Andrew W. Lutz ${ }^{2}$ \\ ${ }^{1}$ Department of Physics, University of Houston-Clear Lake, Houston, TX 77058 \\ ${ }^{2}$ Department of Mathematics, The University of Oklahoma, Norman, OK 73019
}

When supersymmetry breaking is dominated by the complex structure moduli and the universal dilaton, a subset of the supersymmetry parameter space in a realistic MSSM constructed from intersecting/magnetized D-branes are universal, similar to the effective mSUGRA/CMSSM parameter space with a universal scalar mass $m_{0}$, a universal gaugino mass $m_{1 / 2}$ and with the universal trilinear term fixed to be minus the gaugino mass, $A_{0}=-m_{1 / 2}$. More generally, the scalar mass-squared terms for sfermions are split about the Higgs mass-squared terms, $m_{Q_{L}, L_{L}}^{2}=m_{H}^{2}-\Delta m^{2}$ and $m_{Q_{R}, L_{R}}^{2}=m_{H}^{2}+\Delta m^{2}$, for generic values of the Kähler moduli. The scalar masses are universal only for a specific choice of the Kähler moduli. The hyberbolic branch/focus point $(\mathrm{HB} / \mathrm{FP})$ regions of this parameter space are present for both $\Delta m^{2}=0$ and $\Delta m^{2} \neq 0$. Interestingly, it is known that focus points may be realized with any boundary condition of the form $\left(m_{H_{u}}^{2}, m_{U_{3}}^{2}, m_{Q_{3}}^{2}\right) \propto(1,1+x, 1-x)$ with $x$ an arbitrary constant, the same form as those in this model. Thus, we should expect to obtain the same set of focus points in the model regardless of the choice of Kähler moduli. It should be emphasized that the more general choice of Kähler moduli goes beyond mSUGRA/CMSSM. It is shown that there exists superpartner spectra with a light Higgsino-like LSP with $230-350 \mathrm{GeV}$ and a Higgs mass in the range $124-126 \mathrm{GeV}$, and which satisfy most standard experimental constraints. Consequently, viable spectra with low EWFT between $3-7 \%$ may be obtained. The spin-independent direct-detection crosssections are in range of future experiments such as XENON-1T and super CDMS, while the relic density is smaller than the WMAP and Planck bounds by roughly a factor of ten, implying that the LSP is subdominant component of dark matter. In addition, most of the spectra are consistent with constraints from indirect-detection experiments.

PACS numbers: $12.60 . \mathrm{Jv}, 11.25 . \mathrm{Wx}$ 


\section{INTRODUCTION}

String theory is currently one of the most promising frameworks which permits a unification between quantum field theory and general relativity. As such, it should be able to describe the physics of our universe in detail. There are in fact many string theory compactifictions which come very close to reproducing the Standard Model (SM) and its supersymmetric extension, the Minimal Supersymmetric Standard Model (MSSM). Examples include heterotic string compactications on orbifolds [1 3] and on Calabi-Yau manifolds [4, 5], and free fermionic models [6 10]. More, recently compactifications involving D-branes have attracted much attention. These types of models fall into two general classes, intersecting/magnetized D-brane models on orientifold backgrounds [11 21], and Gepner models [22, 23]. One string-derived MSSM satisfying all global consistency conditions has been constructed from intersecting/magnetized D-branes within the context of Type II orientifold compactifications [24 26] on a $T^{6} /\left(\mathbb{Z}_{2} \times \mathbb{Z}_{2}\right)$ background. This model contains three generations of quarks and leptons as well as a single pair of Higgs fields ${ }^{1}$. The model contains a minimal amount of exotic matter, which may be decoupled from the low-energy sector. In addition, the tree-level gauge couplings are automatically unified at the string scale [25, 26]. Thus, this is a phenomenologically interesting model worthy of detailed study.

Supersymmetry (SUSY) and supergravity (SUGRA) are intimately related to string theory. In particular, string theory requires supersymmetry for consistency. Furthermore, supergravity theories arise as the low-energy limits of string theories. Although supersymmetry seems to be a required ingredient of string theory, this does not necessarily imply that it is broken at an energy scale such that we should expect supersymmetric partners of the SM particles to be observed at energies accessible at the Large Hadron Collider (LHC). However, there are many phenomenological reasons to expect this. In particular, SUSY is one of the best-motivated solutions to the hierarchy problem. Although the exact mechanism and scale at which SUSY is broken in nature should it exist is not known, simple calculations suggest that the masses of (at least some) of the superpartners should have $\mathcal{O}(1 \mathrm{TeV})$ masses if SUSY solves the hierarchy problem without introducing any (or not too much) fine-tuning. Moreover, it can be shown that there is an upper bound on the Higgs mass in the MSSM,

${ }^{1}$ Note that the Higgs sector of the model considered in [24] is different than in [25, 26] 
$m_{h} \lesssim 130 \mathrm{GeV}$ [27]. In addition to providing a solution to the hierarchy problem, SUSY with R-parity imposed can provide a natural candidate for dark matter 28 30]. Finally, the unification of the three gauge couplings when extrapolated to high energies via the Renormalization Group Equations (RGE) is much more precise when SUSY is incorporated into the Minimal Supersymmetric Standard Model (MSSM) in comparison to the non-SUSY SM, consistent with the idea of Grand Unification Theories (GUTs) [31, 32].

The discovery of a 124-126 GeV Higgs boson [33, 34] at the (LHC) has so far not been followed by the discovery of any new physics which might explain the hierarchy problem. Supersymmetry remains one of the best candidates for such new physics. However, direct searches during the first run of the LHC for squarks and gluinos are pushing the mass limits for these particles into the $\mathrm{TeV}$ range [35-39]. Furthermore, to obtain a $\sim 125 \mathrm{GeV}$ Higgs mass in the MSSM requires large radiative corrections involving the top/stop sector, requiring large stop squark masses $\mathcal{O}(\mathrm{TeV})$ and/or large values of $\tan \beta$.

The most-studied framework for supersymmetry breaking is minimal supergravity (mSUGRA), or equivalently the Constrained MSSM (CMSSM) [40 42]. However, to obtain a sufficiently large Higgs mass in mSUGRA/CMSSM requires heavy squarks and sleptons which generically spoils the naturalness in which the hierarchy problem is solved by introducing some amount of electroweak fine-tuning (EWFT). One possible exception to this is the hyperbolic branch (HB)/focus point (FP) region of the mSUGRA/CMSSM parameter space characterized by large $m_{0}$ in comparison to $m_{1 / 2}$ where the amount of required EWFT is minimized locally relative to the full parameter space with similar values of $m_{0}$ [43 48]. Several different groups have recently reassessed the status of mSUGRA/CMSSM in light of the $\sim 125 \mathrm{GeV}$ Higgs discovery [49 62] A consensus has built that the mSUGRA/CMSSM parameter space is being strongly constrained by this discovery and pushed into regions which require some degree of fine-tuning [63]. This has motivated the study of extensions of mSUGRA/CMSSM such as the non-universal Higgs mass models (NUHM) where the universality condition on the scalar soft masses of mSUGRA/CMSSM is relaxed such that the Higgs soft masses may be treated as independent parameters [61, 64 67]. This allows superpartner spectra with a $\mu$-parameter close to the electroweak scale, thus featuring low fine-tuning, to be easily obtained. These models generically feature light Higgsinos with masses in the few-hundred $\mathrm{GeV}$ range. More generally, models which feature radiativelydriven natural supersymmetry (RNS) may be considered [68. 
In the phenomenologically interesting intersecting/magnetized D-brane model discussed above, it is possible to study the possible sets of supersupersymmetric soft terms which may be obtained, specifically for the F-type supersymmetry breaking where SUSY-breaking is dominated by the complex structure and/or Kähler moduli as well as the universal dilaton. In general, the sets of possible soft terms in the model are non-universal. Investigations into the sets of non-universal soft terms have been performed in [25, 26, 69, 70]. However, there also exists subsets of the soft terms which feature universality. As shown in [24], these subsets are similar to the the effective mSUGRA/CMSSM parameter space in that they feature a universal scalar mass $m_{0}$, a universal gaugino mass $m_{1 / 2}$ and a universal trilinear term $A_{0}$, which for the present case is fixed such that $A_{0}=-m_{1 / 2}$. These boundary conditions coincide with the slice of the effective mSUGRA/CMSSM parameter space studied in [74], which includes a band along the HB/FP branch where spectra with a Higgs mass in the range $124-126 \mathrm{GeV}$ may be obtained in addition to sastifying experimental bounds on the dark matter relic density while also satisying most standard experimental constraints. In addition, for generic values of the Kähler moduli in the model, the soft terms for sfermions are split about the Higgs mass-squared terms as $m_{Q_{L}, L_{L}}^{2}=m_{H}^{2}-\Delta m^{2}$ and $m_{Q_{R}, L_{R}}^{2}=m_{H}^{2}+\Delta m^{2}$ where $\Delta m^{2}$ is a function of the Kähler moduli. Only for a specific choice of the Kähler moduli do the soft terms reduce to those with a universal scalar mass for both Higgs and sfermions. Interestingly, it was observed in [45] that focus points may be realized with any boundary condition of the form $\left(m_{H_{u}}^{2}, m_{U_{3}}^{2}, m_{Q_{3}}^{2}\right) \propto(1,1+x, 1-x)$ with $x$ an arbitrary constant. These are essentially the same as boundary conditions in the model that we are considering, the only difference being that the soft terms in the model have this form for all three families of sfermions. Thus, we should expect to obtain the same set of focus points in the model regardless of the choice of Kähler moduli. It should be emphasized that the more general choice of Kähler moduli goes beyond mSUGRA/CMSSM.

In the following, detailed scans of the D-brane model supersymetry parameter space with $A_{0}=-m_{1 / 2}$ have been performed. Regions of this parameter space featuring light Higgsinos, $\mathrm{a} \sim 125 \mathrm{GeV}$ Higgs mass, and low EWFT are identified and discussed. In particular, it is found that there exists superpartner spectra with a light Higgsino-like LSP with 225 $350 \mathrm{GeV}$ masses and a Higgs mass in the range $124-126 \mathrm{GeV}$, and which satisfy most standard experimental constraints. Consequently, viable spectra with low EWFT between 3-7\% may be obtained. The dark matter direct-detection cross-sections for these spectra are 
in range of future experiments such as XENON-1T [71] and super CDMS [72]. In addition, most of the spectra are consistent with constraints from indirect-detection experiments. Finally, the parameter space where the scalar mass-squared terms for squarks and sleptons are split about the Higgs mass-squared term, $m_{Q_{L}, L_{L}}^{2}=m_{H}^{2}-\Delta m^{2}$ and $m_{Q_{R}, L_{R}}^{2}=m_{H}^{2}+$ $\Delta m^{2}$, is also studied in detail. As mentioned, this parameter space is obtained from the string model for generic values of the Kähler moduli and goes beyond mSUGRA/CMSSM. As expected, the HB/FP regions are also present for this region of the parameter space, independent of the choice of Kähler moduli.

\section{HIGH SCALE BOUNDARY CONDITIONS}

Let us consider the realistic MSSM constructed from interesecting/magnetized D-branes studied in [24]. This simple and elegant model has many desirable phenomenological features such as three families of quarks and leptons, minimal exotic matter, gauge coupling unification, and rank-3 Yukawa mass matrices [25, 26] such that it is possible to obtain the correct fermion masses and mixings. In addition, one variation of the model allows either baryon or lepton number to exists as a local gauge symmetry, thus forbidding proton decay [73]. The model also satisfies all conditions for global consistency such as tadpole and anomaly cancellation.

Type II orientifold string compactifications with intersecting/magnetized D-branes have provided useful geometric tools with which the MSSM may be engineered [21, 76]. To briefly give an over view of the construction of such models, D6-branes in Type IIA fill $(3+1)$-dimensional Minkowski spacetime and wrap 3-cycles in the compactified manifold, such that a stack of $N$ branes generates a gauge group $\mathrm{U}(N)$ [or $\mathrm{U}(N / 2)$ in the case of $\left.T^{6} /\left(\mathbb{Z}_{2} \times \mathbb{Z}_{2}\right)\right]$ in its world volume. In general, the 3-cycles wrapped by the stacks of D6branes intersect multiple times in the internal space, resulting in a chiral fermion in the bifundamental representation localized at the intersection between different stacks $a$ and $b$. The multiplicity of such fermions is then given by the number of times the 3-cycles intersect. Each stack of D6-branes a may intersect the orientifold images of other stacks $b^{\prime}$, also resulting in fermions in bifundamental representations. Each stack may also intersect its own image $a^{\prime}$, resulting in chiral fermions in the symmetric and antisymmetric representations. In addition, the consistency of the model requires certain constraints to be satisfied, namely, 
Ramond-Ramond (R-R) tadpole cancellation and the preservation of $\mathcal{N}=1$ supersymmetry.

The model discussed in [24] describes a three-generation Pati-Salam model with additional hidden sectors. The full gauge symmetry of the model is given by $\left[\mathrm{U}(4)_{C} \times \mathrm{U}(2)_{L} \times\right.$ $\left.\mathrm{U}(2)_{R}\right]_{\text {observable }} \times\left[\mathrm{USp}(2)^{4}\right]_{\text {hidden. }}$. As discussed in detail in [25, 26], with this configuration of D6 branes all R-R tadpoles are canceled, K-theory constraints are satisfied, and $\mathcal{N}=1$ supersymmetry is preserved. Using the effective scalar potential it is possible to study the stability [13], the tree-level gauge couplings [77 79], gauge threshold corrections [80], and gauge coupling unification [81]. The effective Yukawa couplings [82, 83, matter field Kähler metric and soft-SUSY breaking terms have also been investigated [84]. A more detailed discussion of the Kähler metric and string scattering of gauge, matter, and moduli fields has been performed in [85].

The $\mathcal{N}=1$ supergravity action depends upon three functions, the holomorphic gauge kinetic function, $f$, Kähler potential $K$, and the superpotential $W$. Each of these will in turn depend upon the moduli fields which describe the background upon which the model is constructed. Supersymmetry is broken when some of the F-terms of the hidden sector fields $M$ acquire VEVs. This then results in soft terms being generated in the observable sector. For simplicity, it is assumed in this analysis that the $D$-term does not contribute (see [86]) to the SUSY breaking. Then, the goldstino is eaten by the gravitino via the superHiggs effect and thus the gravitino obtains a mass, $m_{3 / 2}$.

The normalized gaugino mass parameters, scalar mass-squared parameters, and trilinear parameters respectively may be given in terms of the Kähler potential, the gauge kinetic function, and the superpotential as

$$
\begin{aligned}
M_{P} & =\frac{1}{2 \operatorname{Re} f_{P}}\left(F^{M} \partial_{M} f_{P}\right) \\
m_{P Q}^{2} & =\left(m_{3 / 2}^{2}+V_{0}\right)-\sum_{M, N} \bar{F}^{M} F^{N} \partial_{\bar{M}} \partial_{N} \log \left(\tilde{K}_{P Q}\right), \\
A_{P Q R} & =F^{M}\left[\hat{K}_{M}+\partial_{M} \log \left(Y_{P Q R}\right)-\partial_{M} \log \left(\tilde{K}_{P Q} \tilde{K}_{Q R} \tilde{K}_{R P}\right)\right],
\end{aligned}
$$

where $\tilde{K}_{Q R}$ is the Kähler metric appropriate for D-branes which are parallel on at least one torus, i.e. involving non-chiral matter. We assume that the supersymmetric breaking in the model is dominated by the complex structure moduli $u^{i}, i=1,2,3$ which describe the shape of the compactified dimensions as well as the universal dilaton $s$. We allow the dilaton $s$ and the $u$-moduli to obtain vacuum expectation values (VEVs). To do this, we parameterize the 
$F$-terms as

$$
F^{u^{i}, s}=\sqrt{3} m_{3 / 2}\left[(s+\bar{s}) \Theta_{s}+\left(u^{i}+\bar{u}^{i}\right) \Theta_{i}^{u}+\left(t^{i}+\bar{t}^{i}\right) \Theta_{i}^{t}\right]
$$

The parameters $\Theta_{i}$ parameterize the goldstino direction in $U^{i}$ space, where $\sum\left(\left|\Theta_{i}^{u}\right|^{2}+\left|\Theta_{s}\right|^{2}=\right.$ 1. The goldstino angle $\Theta_{s}$ determines the degree to which SUSY breaking is being dominated by the dilaton $s$ and/or complex structure $\left(u^{i}\right)$ and Kähler $\left(t^{i}\right)$ moduli. For the present, we do not allow the Kähler moduli $t^{i}$ to obtain VEVs so that $\Theta_{i}^{t}=0$.

In general, the soft terms for intersecting D-brane models are non-universal. However, universal soft terms may arise in the model under study for the case $\Theta_{1}^{u}=\Theta_{2}^{u} \equiv \Theta_{12}$ and $\Theta_{3}^{u}=\Theta_{S} \equiv \Theta_{3 s}$. We refer the reader to ref. [24] for a detailed derivation of the resulting soft terms. For convenience, let us define $\Theta_{12}=\frac{1}{\sqrt{2}} \cos \theta$ and $\Theta_{3 s}=\frac{1}{\sqrt{2}} \sin \theta$. Then, the soft terms take the simple form

$$
\begin{gathered}
m_{H}^{2}=\frac{m_{3 / 2}^{2}}{4} \\
m_{1 / 2}=\sqrt{\frac{3}{2}} m_{H}[1+\sin (2 \theta)]^{1 / 2} \\
m_{Q_{L}, L_{L}}^{2}=m_{H}^{2}-\Delta m^{2}, \\
m_{Q_{R}, L_{R}}^{2}=m_{H}^{2}+\Delta m^{2}, \\
A_{0}=-m_{1 / 2},
\end{gathered}
$$

with

$$
\Delta m^{2}=\frac{6 m_{H}^{2}}{\pi}\left[\cos ^{2} \theta-\sin ^{2} \theta\right] \psi(t, \bar{t})
$$

where $\psi(t, \bar{t})$ is a function which depends on the Kähler moduli, as shown in [24] and $m_{3 / 2}$ is the gravitino mass.

There are two cases of special note where $\Delta m^{2}=0$, resulting in a universal scalar mass. First, taking $\theta=\pi / 4$ the soft terms become those of the special dilaton solution,

$$
m_{1 / 2}=\frac{\sqrt{3}}{2} m_{3 / 2}, \quad m_{0}=\frac{m_{3 / 2}}{2}, \quad A_{0}=-m_{1 / 2},
$$




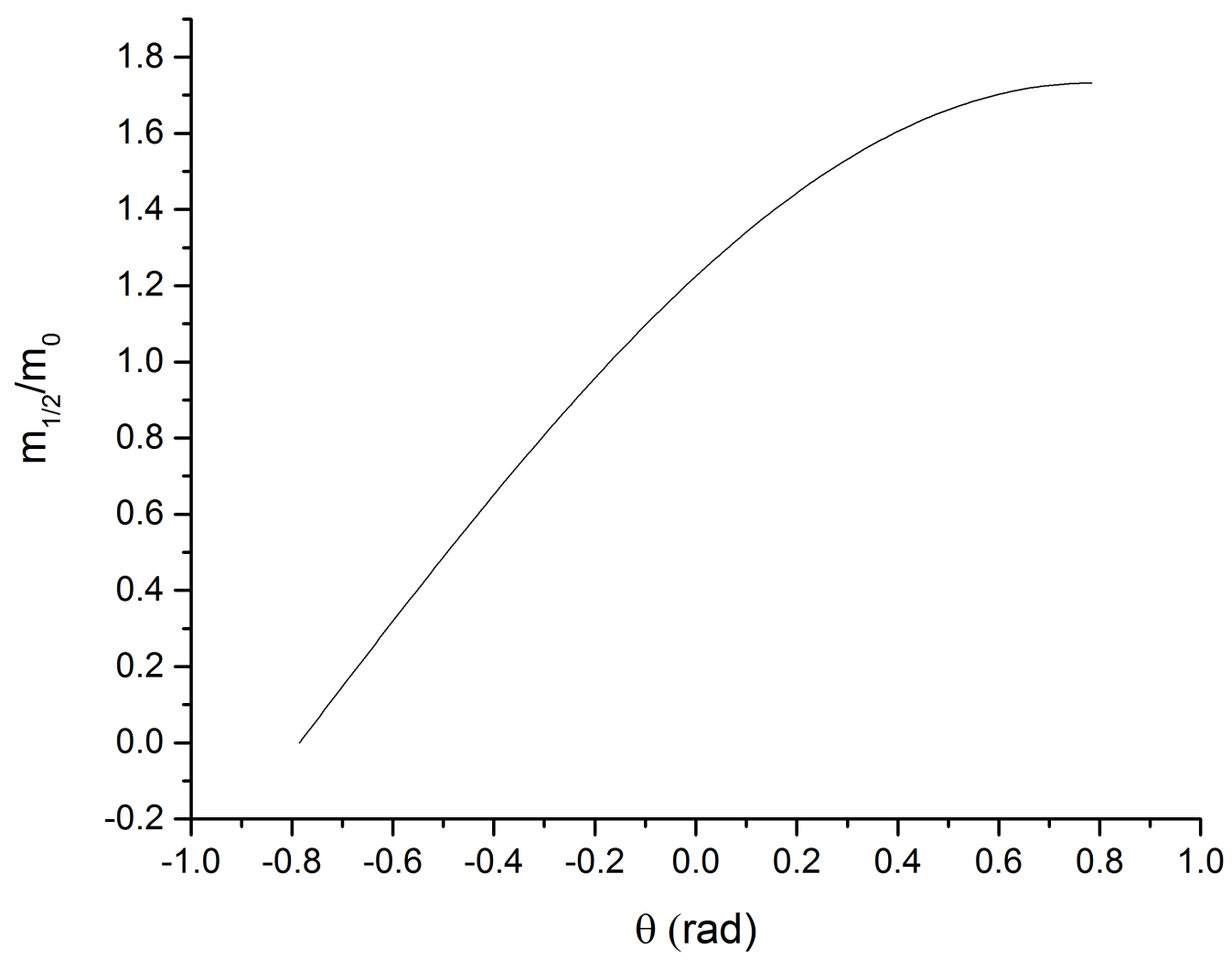

FIG. 1: Gaugino mass to scalar mass ratio as a function of $\theta$ for $\Delta m^{2}=0$, i.e. $\operatorname{mSUGRA} / \mathrm{CMSSM}$ with $A_{0}=-m_{1 / 2}$.

which is well-known from heterotic string compactifications. In fact, these boundary conditions are generic for all Pati-Salam models constructed from intersecting D-branes as shown in [24]. A recent study of this parameter space has been performed [87, 88]. The second case where $\Delta m^{2}=0$ is for a specific value of the Kähler moduli as shown in [24]. The parameter space for this scenario essentially is similar to the case of mSUGRA/CMSSM in that it features a universal scalar mass $m_{0}$, a universal gaugino mass $m_{1 / 2}$ and a universal trilinear term which is fixed as $A_{0}=-m_{1 / 2}$ for the present case. For more general values of the Kähler moduli, the scalar masses-squared for sfermions are split about the Higgs scalar mass-squared. In principle, it should be possible to dynamically fix the Kähler moduli, complex structure moduli and the dilaton in the model by including non-perturbative effects such as supergravity fluxes or gaugino condensation in a hidden sector. However, for the 


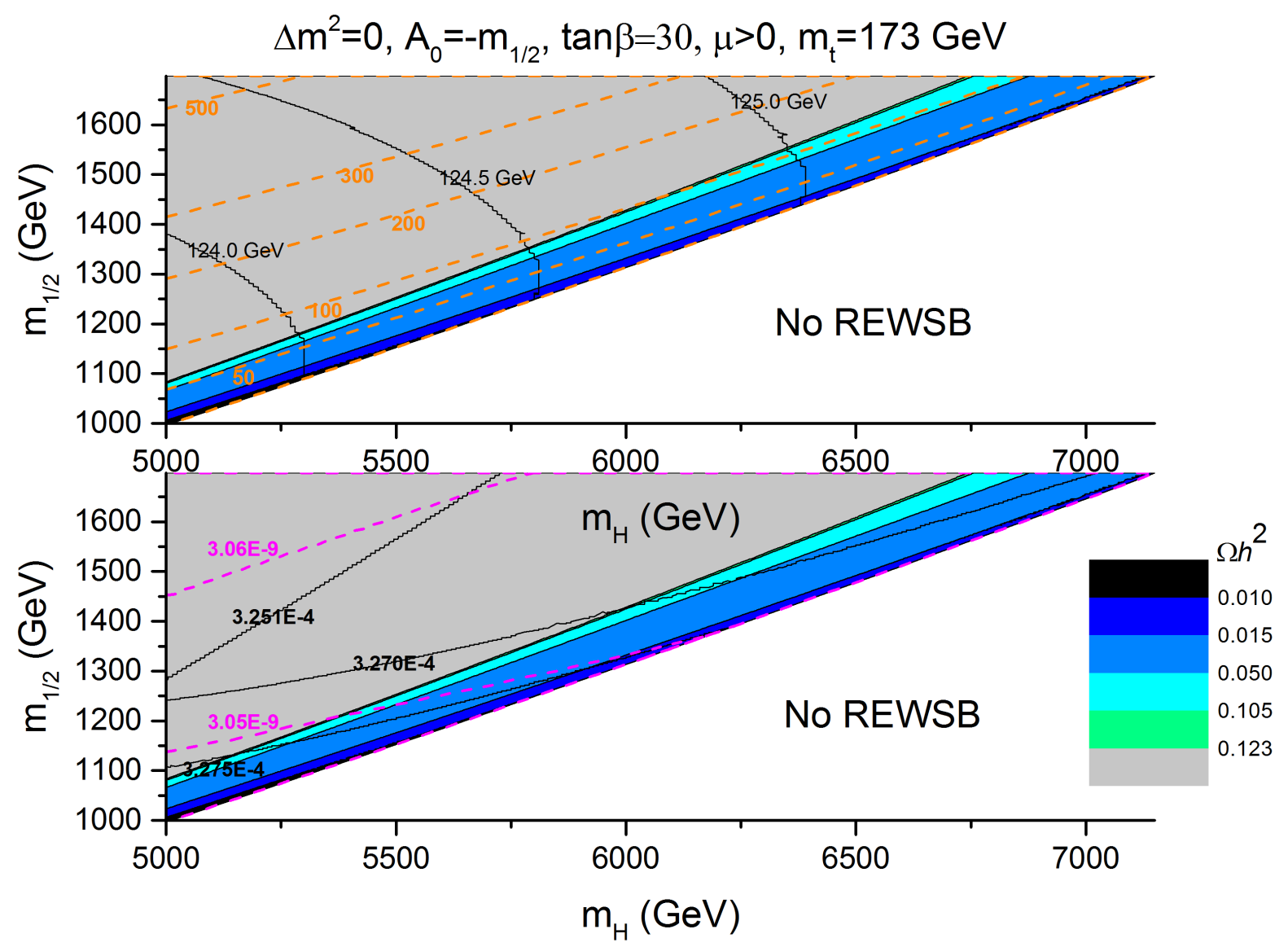

FIG. 2: The $m_{1 / 2}$ vs. $m_{H}=m_{0}$ plane with $\Delta m^{2}=0, A_{0}=-m_{1 / 2}, \mu>0, \tan \beta=30$, and $m_{t}=173 \mathrm{GeV}$. The regions shaded in dark blue indicate $\Omega_{\chi^{0}} h^{2} \lesssim 0.123$, while the region shaded in green satisfies the WMAP 9-year $2 \sigma$ bounds, $0.105 \lesssim \Omega_{\chi^{0}} h^{2} \lesssim 0.123$. Top panel: Values of the higgs mass $m_{h}$ are denoted by solid black contours while dashed orange contours indicate values for minimal electroweak fine-tuning, $\Delta_{E W}$. Bottom panel: Values for the Flavor Changing Neutral Current (FCNC) process, $b \rightarrow s \gamma$ are indicated by solid black contours, while dashed magenta contours indicate values for the process $B_{s}^{0} \rightarrow \mu^{+} \mu^{-}$.

present we shall treat the Kähler moduli as free parameters, and there is no gaurantee that they will take the special case noted above where $\Delta m^{2}=0$. Our objective in the following sections is thus to study the more general parameter space with $\Delta m^{2} \neq 0$, which occurs for generic values of the Kähler moduli, in addition to the case with $\Delta m^{2}=0$.

As noted in the Introduction, it was observed in [45] that focus points may be realized with any boundary condition of the form $\left(m_{H_{u}}^{2}, m_{U_{3}}^{2}, m_{Q_{3}}^{2}\right) \propto(1,1+x, 1-x)$ with $x$ an 


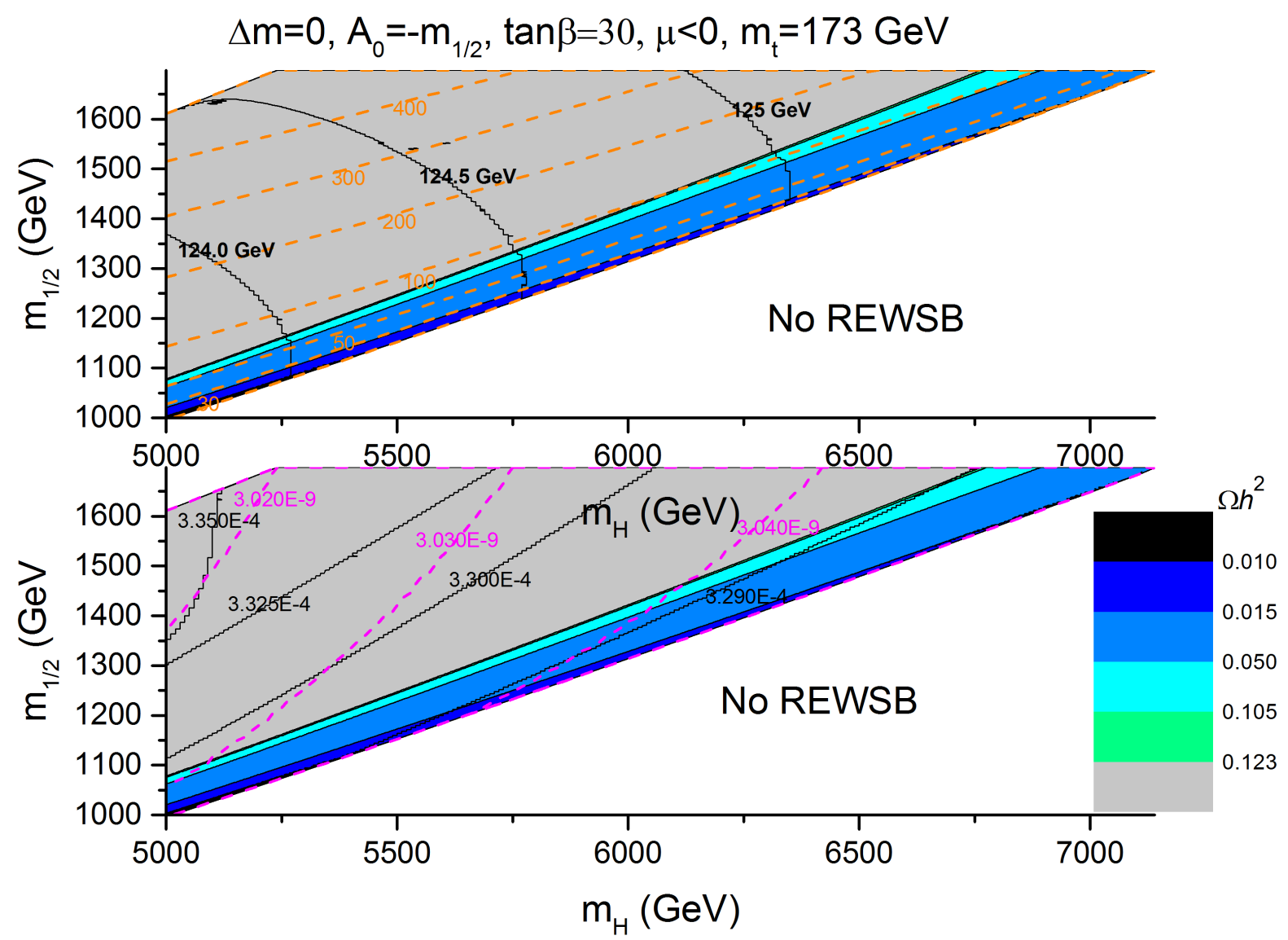

FIG. 3: The $m_{1 / 2}$ vs. $m_{H}=m_{0}$ plane with $\Delta m^{2}=0, A_{0}=-m_{1 / 2}, \mu<0, \tan \beta=30$, and $m_{t}=173 \mathrm{GeV}$. The regions shaded in dark blue indicate $\Omega_{\chi^{0}} h^{2} \lesssim 0.123$, while the region shaded in green satisfies the WMAP 9-year $2 \sigma$ bounds, $0.105 \lesssim \Omega_{\chi^{0}} h^{2} \lesssim 0.123$. Top panel: Values of the higgs mass $m_{h}$ are denoted by solid black contours while dashed orange contours indicate values for minimal electroweak fine-tuning, $\Delta_{E W}$. Bottom panel: Values for the Flavor Changing Neutral Current (FCNC) process, $b \rightarrow s \gamma$ are indicated by solid black contours, while dashed magenta contours indicate values for the process $B_{s}^{0} \rightarrow \mu^{+} \mu^{-}$.

arbitrary constant. The above soft terms have exactly this form with $\Delta m^{2}=x$. Thus, we should find that a focus point in the parameter space with $\Delta m^{2}=0$ is also a focus point when $\Delta m^{2} \neq 0$. Since $\Delta m^{2}$ is a function which depends on the Kähler moduli, it should be the case that the $\mathrm{HB} / \mathrm{FP}$ region of the parameter space is essentially independent of the Kähler moduli. Indeed this is the case, as we shall see. 


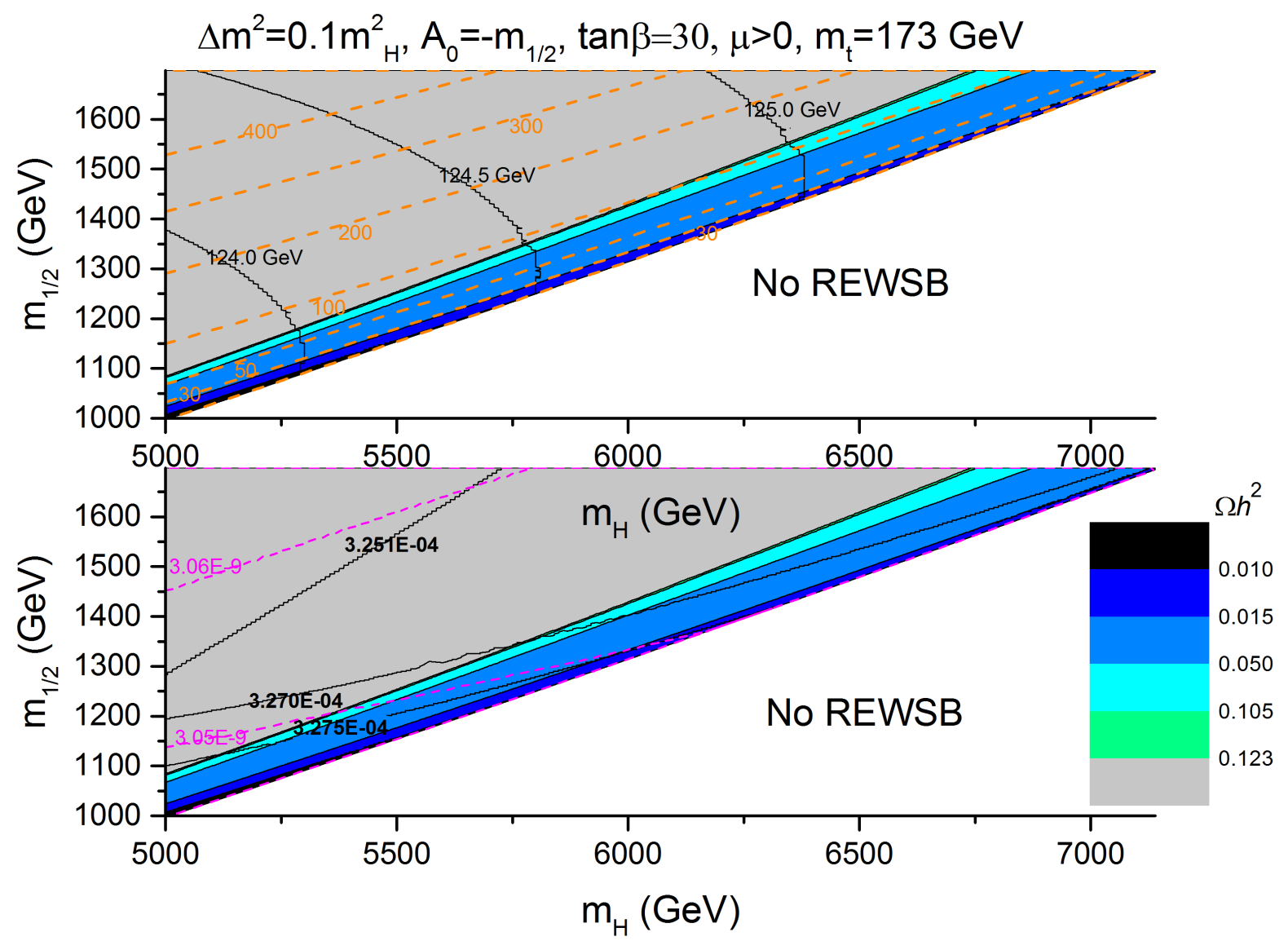

FIG. 4: The $m_{1 / 2}$ vs. $m_{H}=m_{0}$ plane with $\Delta m^{2}=0.1 m_{H}^{2}, A_{0}=-m_{1 / 2}, \mu>0, \tan \beta=30$, and $m_{t}=173 \mathrm{GeV}$. The regions shaded in dark blue indicate $\Omega_{\chi^{0}} h^{2} \lesssim 0.123$, while the region shaded in green satisfies the WMAP 9-year $2 \sigma$ bounds, $0.105 \lesssim \Omega_{\chi^{0}} h^{2} \lesssim 0.123$. Top panel: Values of the higgs mass $m_{h}$ are denoted by solid black contours while dashed orange contours indicate values for minimal electroweak fine-tuning, $\Delta_{E W}$. Bottom panel: Values for the Flavor Changing Neutral Current (FCNC) process, $b \rightarrow s \gamma$ are indicated by solid black contours, while dashed magenta contours indicate values for the process $B_{s}^{0} \rightarrow \mu^{+} \mu^{-}$.

\section{PARAMETER SPACE}

As noted in the previous section, the high-scale boundary conditions for this model are universal for a specific value of the Kähler moduli. For this case the soft terms are similar to those of the Constrained Minimal Supersymmetric Standard Model (CMSSM), one of the most studied model of supersymmetry breaking. These models are characterized by 


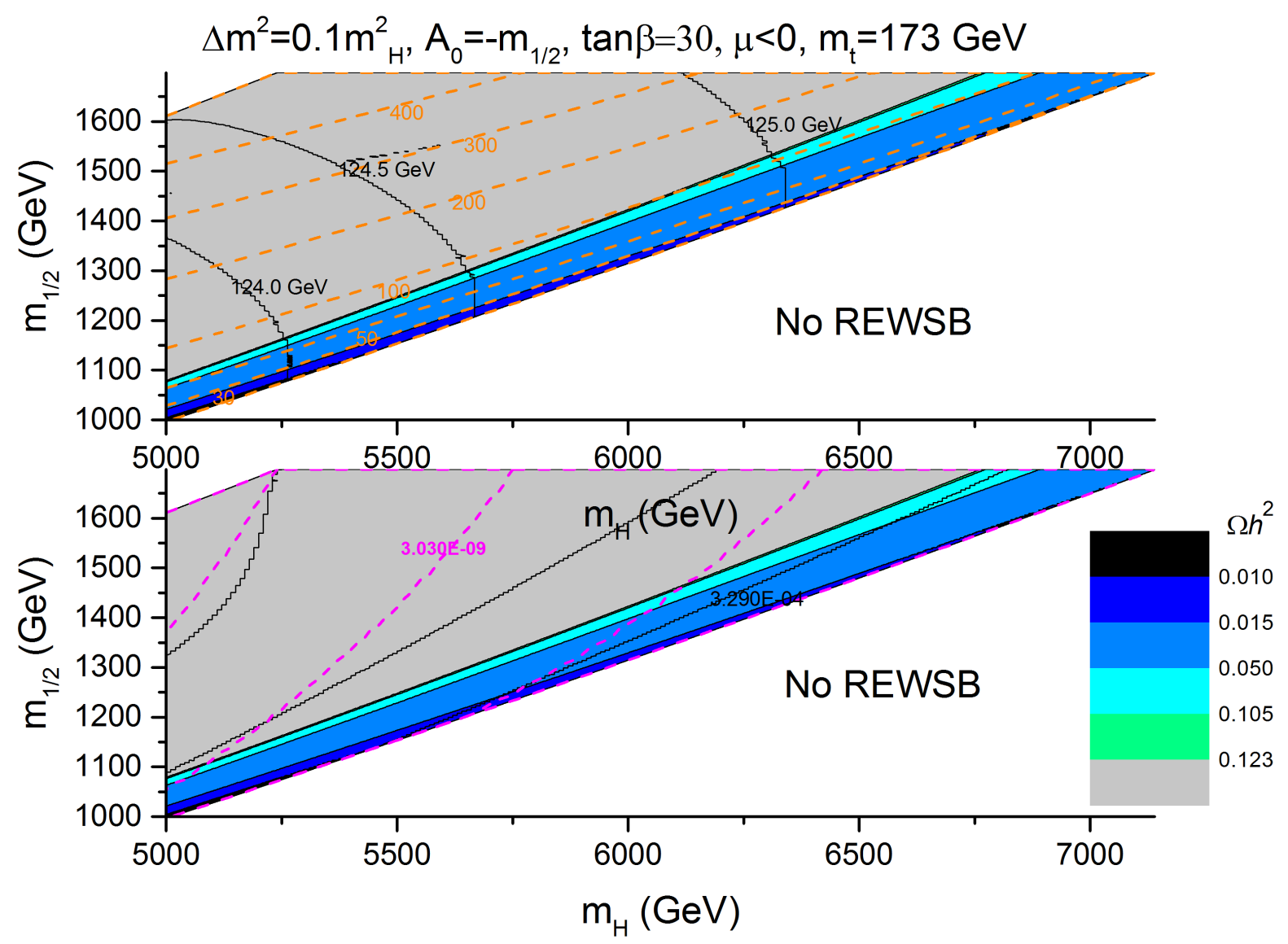

FIG. 5: The $m_{1 / 2}$ vs. $m_{H}=m_{0}$ plane with $\Delta m^{2}=0.1 m_{H}^{2}, A_{0}=-m_{1 / 2}, \mu<0, \tan \beta=30$, and $m_{t}=173 \mathrm{GeV}$. The regions shaded in dark blue indicate $\Omega_{\chi^{0}} h^{2} \lesssim 0.123$, while the region shaded in green satisfies the WMAP 9-year $2 \sigma$ bounds, $0.105 \lesssim \Omega_{\chi^{0}} h^{2} \lesssim 0.123$. Top panel: Values of the higgs mass $m_{h}$ are denoted by solid black contours while dashed orange contours indicate values for minimal electroweak fine-tuning, $\Delta_{E W}$. Bottom panel: Values for the Flavor Changing Neutral Current (FCNC) process, $b \rightarrow s \gamma$ are indicated by solid black contours, while dashed magenta contours indicate values for the process $B_{s}^{0} \rightarrow \mu^{+} \mu^{-}$.

the following parameters: a universal scalar mass $m_{0}$, a universal gaugino mass $m_{1 / 2}$, the Higgsino mixing $\mu$-parameter, the Higgs bilinear $B$-parameter, a universal trilinear coupling $A_{0}$, and $\tan \beta$. One then determines the $B$ and $|\mu|$ parameters by the minimization of the Higgs potential triggering REWSB [89, 90, with the sign of $\mu$ remaining undetermined. One is then left with the following free parameters at the GUT scale: $m_{0}, m_{1 / 2}, A_{0}, \tan \beta$ and $\operatorname{sign}(\mu)$. For the string-derived model of the previous section, we may also trade $B$ and 


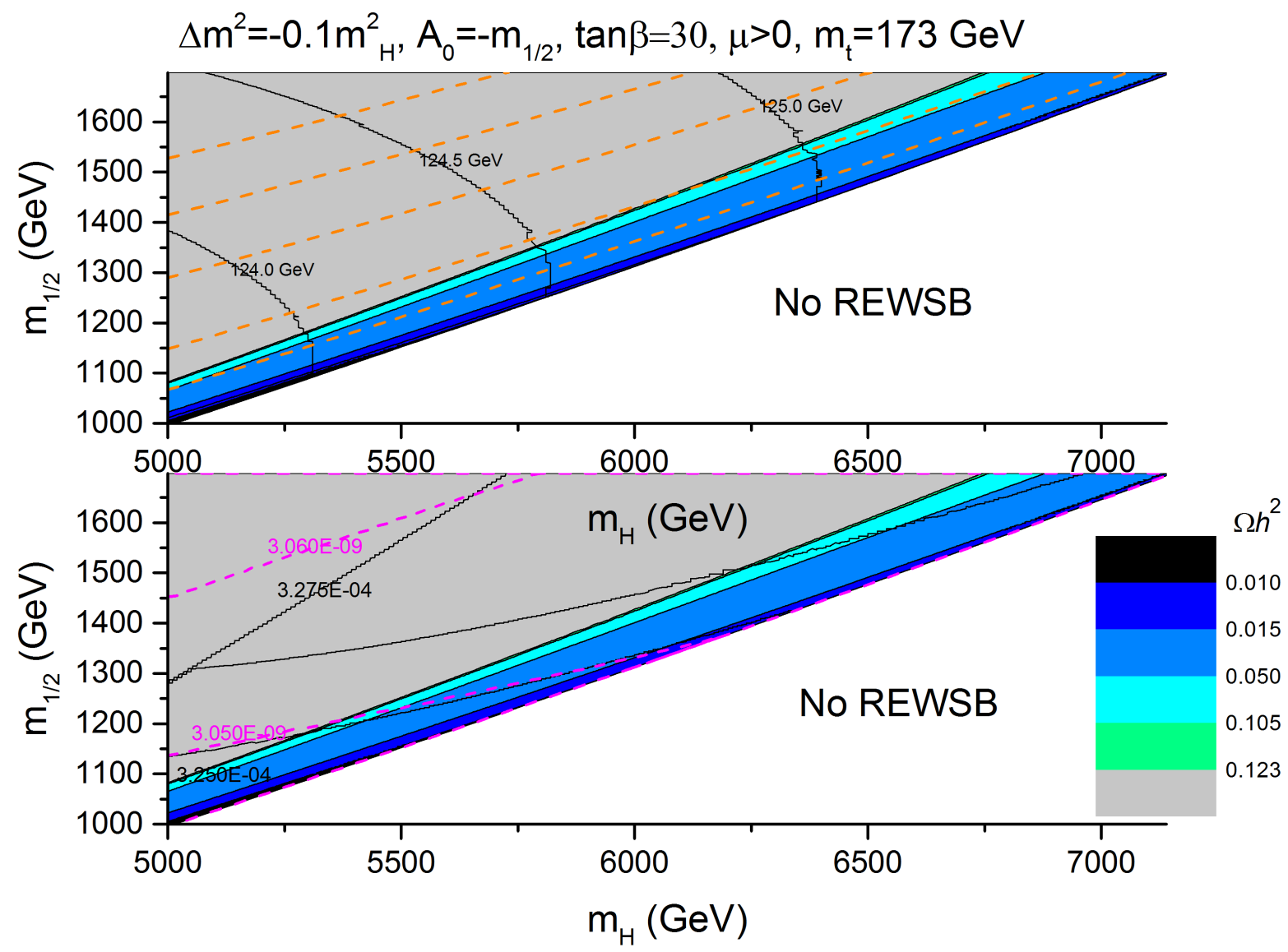

FIG. 6: The $m_{1 / 2}$ vs. $m_{H}=m_{0}$ plane with $\Delta m^{2}=-0.1 m_{H}^{2}, A_{0}=-m_{1 / 2}, \mu>0, \tan \beta=30$, and $m_{t}=173 \mathrm{GeV}$. The regions shaded in dark blue indicate $\Omega_{\chi^{0}} h^{2} \lesssim 0.123$, while the region shaded in green satisfies the WMAP 9-year $2 \sigma$ bounds, $0.105 \lesssim \Omega_{\chi^{0}} h^{2} \lesssim 0.123$. Top panel: Values of the higgs mass $m_{h}$ are denoted by solid black contours while dashed orange contours indicate values for minimal electroweak fine-tuning, $\Delta_{E W}$. Bottom panel: Values for the Flavor Changing Neutral Current (FCNC) process, $b \rightarrow s \gamma$ are indicated by solid black contours, while dashed magenta contours indicate values for the process $B_{s}^{0} \rightarrow \mu^{+} \mu^{-}$.

$\mu$ for $\tan \beta$ and $\operatorname{sign}(\mu)$ so that we have effectively the same set of GUT scale parameters as for the CMSSM, with the trilinear terms related to the gaugino mass as $A_{0}=-m_{1 / 2}$. However, it should be kept in mind that the truly fundamental parameters are $m_{1 / 2}, \theta, B$ and $\mu$ from which the effective parameters $m_{0}, m_{1 / 2}$, and $A_{0}$ are related by Eqns. 7 . In addition, the sfermion mass splitting $\Delta m$ depends upon the Kähler moduli of the stringderived model. This splitting is zero for a specific value of the Kähler moduli, and so the 


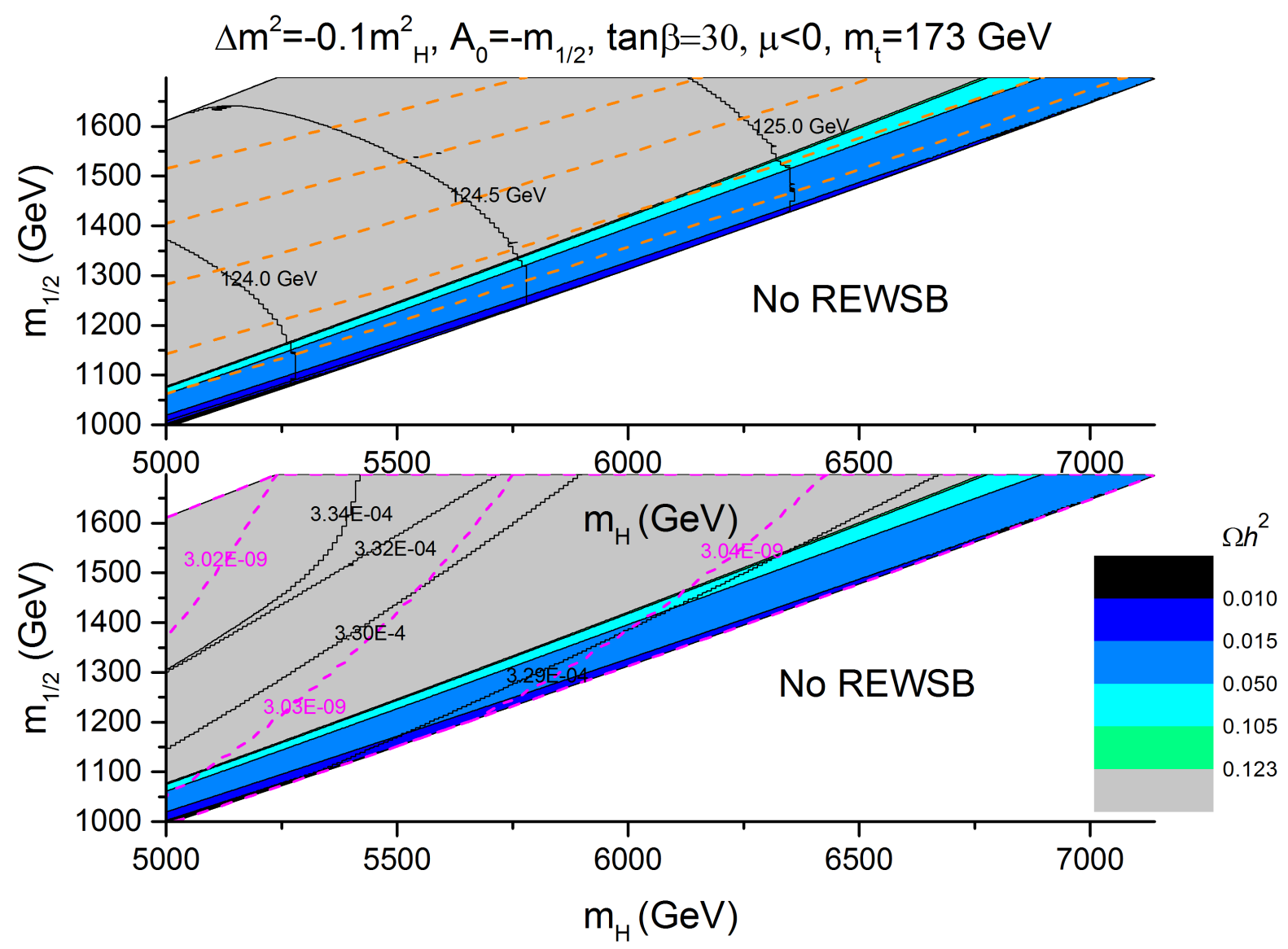

FIG. 7: The $m_{1 / 2}$ vs. $m_{H}=m_{0}$ plane with $\Delta m^{2}=-0.1 m_{H}^{2}, A_{0}=-m_{1 / 2}, \mu<0, \tan \beta=30$, and $m_{t}=173 \mathrm{GeV}$. The regions shaded in dark blue indicate $\Omega_{\chi^{0}} h^{2} \lesssim 0.123$, while the region shaded in green satisfies the WMAP 9-year $2 \sigma$ bounds, $0.105 \lesssim \Omega_{\chi^{0}} h^{2} \lesssim 0.123$. Top panel: Values of the higgs mass $m_{h}$ are denoted by solid black contours while dashed orange contours indicate values for minimal electroweak fine-tuning, $\Delta_{E W}$. Bottom panel: Values for the Flavor Changing Neutral Current (FCNC) process, $b \rightarrow s \gamma$ are indicated by solid black contours, while dashed magenta contours indicate values for the process $B_{s}^{0} \rightarrow \mu^{+} \mu^{-}$.

GUT-scale parameters are universal for this case. In the following, we will study the resulting supersymmetry parameter space for both $\Delta m=0$ and $\Delta m \neq 0$.

The high-scale boundary conditions derived from the model are input into suSpect 2.41 91] to evolve the soft terms down to the electroweak scale via the Renormalization Group Equations (RGEs) and then input into MicrOMEGAs 3.6.9.2 [92 94] to calculate the corresponding relic neutralino density, direct-detection cross-sections, and indirect-detection 
annihilation cross-sections. In particular, we shall focus on the $\mathrm{FP} / \mathrm{HB}$ region of the parameter space. We vary $m_{0}$ (or equivalently $m_{H}$ for the cases with split sfermion masses) in increments of $2 \mathrm{GeV}$ between $5000-7500 \mathrm{GeV}$ and $m_{1 / 2}$ in increments of $1 \mathrm{GeV}$ for each scan. We fix $\tan \beta=30$ for convenience, although our results are generic to other values of $\tan \beta$. The Higgsino mass parameter $\mu$ is determined by the requirement of radiative electroweak symmetry breaking (REWSB). In addition to imposing experimental constraints, the spectra are filtered from the final data set if the iterative procedure employed by SuSpect does not converge to a reliable solution. We take the top quark mass to be $m_{t}=173.3 \pm 1.1 \mathrm{GeV}$ [95]. We do not fix the sign of $\mu$ as the contribution to $g_{\mu}-2$ for the muon is small for superpartner spectra with heavy scalars. In analyzing the resulting data, we consider the following experimental constraints:

1. The first results from the Planck experiment [96], with a dark matter density in the range $\Omega_{c} h^{2}=0.1199 \pm 0.0027$ and the WMAP 9-year $2 \sigma$ preferred range [97] for the cold dark matter density, $0.105 \leq \Omega_{\chi^{o}} h^{2} \leq 0.123$. We consider two cases, one where a neutralino LSP is the dominant component of the dark matter and another where it makes up a subdominant component such that $0 \leq \Omega_{\chi^{\circ}} h^{2} \leq 0.123$.

2. The experimental limits on the Flavor Changing Neutral Current (FCNC) process, $b \rightarrow s \gamma$. The results from the Heavy Flavor Averaging Group (HFAG) [98], in addition to the BABAR, Belle, and CLEO results, are: $\operatorname{Br}(b \rightarrow s \gamma)=\left(355 \pm 24_{-10}^{+9} \pm 3\right) \times 10^{-6}$. There is also a more recent estimate [99] of $\operatorname{Br}(b \rightarrow s \gamma)=(3.15 \pm 0.23) \times 10^{-4}$. For our analysis, we use the limits $2.86 \times 10^{-4} \leq \operatorname{Br}(b \rightarrow s \gamma) \leq 4.18 \times 10^{-4}$, where experimental and theoretical errors are added in quadrature.

3. The process $B_{s}^{0} \rightarrow \mu^{+} \mu^{-}$which has recently been observed to be in the range $2 \times 10^{-9}<$ $B F\left(B_{s}^{0} \rightarrow \mu^{+} \mu^{-}\right)<4.7 \times 10^{-9}$ by LHCb [100].

4. The lightest $\mathrm{CP}$-even Higgs mass in the range $124 \mathrm{GeV} \lesssim m_{h} \lesssim 126 \mathrm{GeV}$ as observed by the ATLAS and CMS experiments at the LHC [33, 34].

We will not require that the problem of the anomalous magnetic moment of the muon [101], $4.7 \times 10^{-10} \leq a_{\mu} \approx 52.7 \times 10^{-10}$, is solved by contributions from supersymmetric particles as the spectra that will be studied may only make small contributions. 

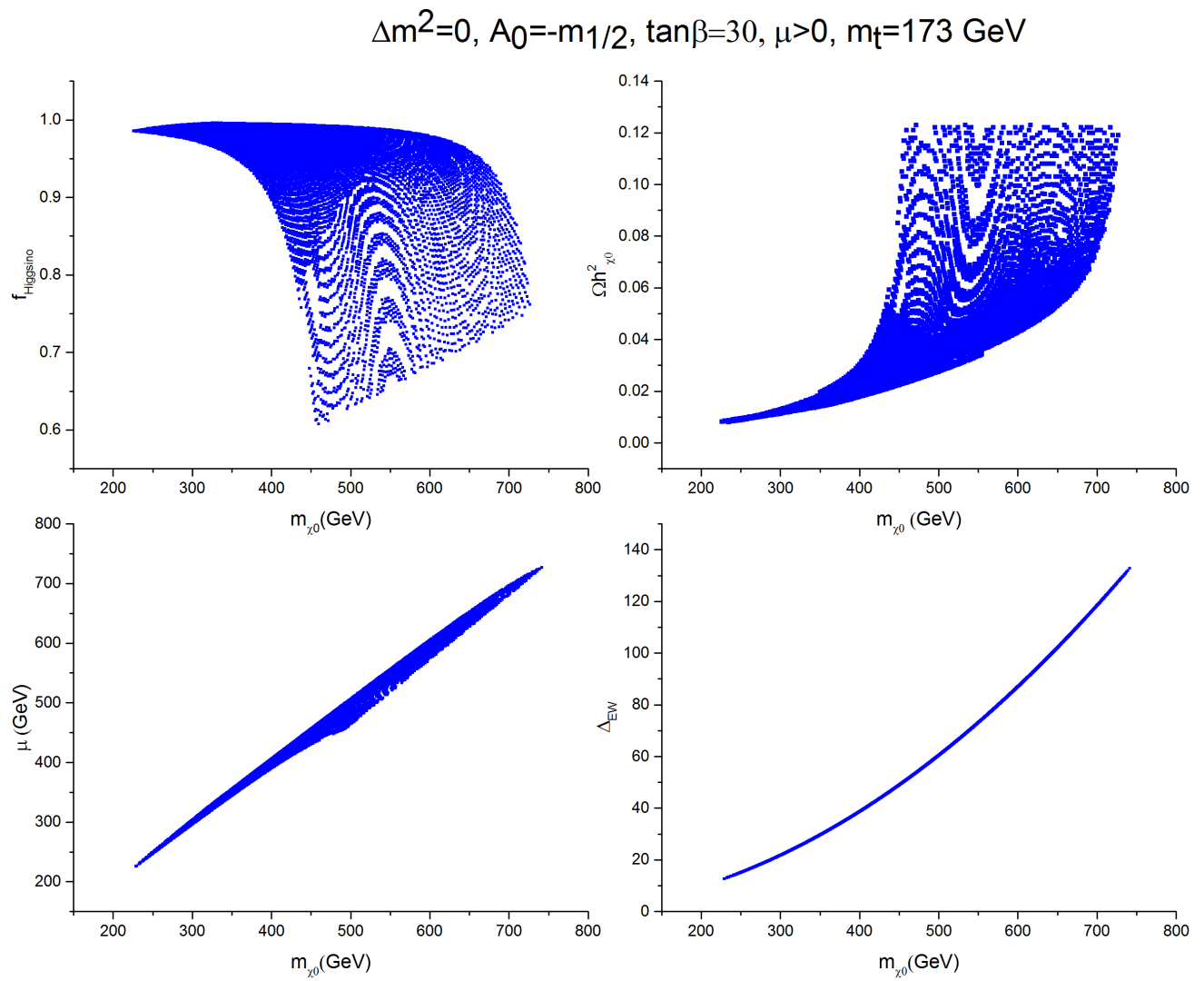

FIG. 8: Top left panel: Higgsino fraction vs. LSP mass. Top right panel: Relic density vs. LSP mass. Bottom left panel: Higgsino bilinear $\mu$ vs. LSP mass $m_{\tilde{\chi}^{0}}$. Bottom right panel: Minimal electroweak fine-tuning $\Delta_{E W}$ vs. LSP mass. For each plot, $m_{\tilde{\chi}^{0}}$ for $\Delta m^{2}=0, \tan \beta=30, \mu>0$, and $m_{t}=173 \mathrm{GeV}$.

Furtheremore, there are large hadronic contributions to this anomaly that require delicate subtractions with large uncertainties [102].

One of the most compelling motivations for introducing low-scale SUSY is to solve the hierarchy problem. An important question is whether or not this is accomplished naturally without reintroducing any fine-tuning. In fact, the mass of the observed Higgs boson is slightly too large to accomodate in models such as mSUGRA/CMSSM without some level of fine-tuning. This is refererred to as the little hierarchy problem. Ordinarily, spectra with large scalar masses would generically be considered highly fine-tuned. This is not necessarily true for those spectra which fall in the $\mathrm{HB} / \mathrm{FP}$ region of the parameter space, which may 

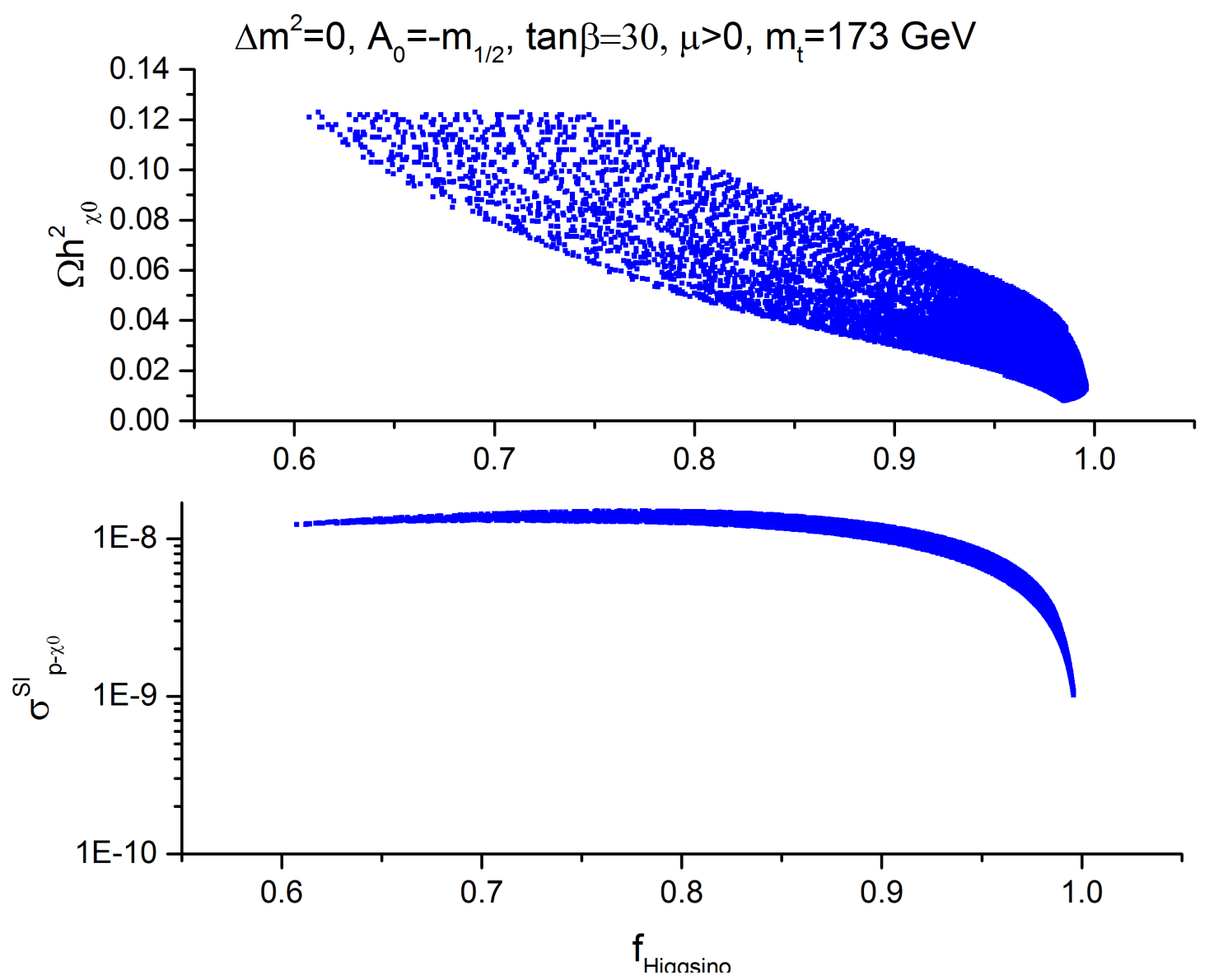

FIG. 9: Top panel: LSP relic density $\Omega_{\tilde{\chi}^{0}}$ vs. higgsino fraction $f_{\text {higgsino }}$ for $\Delta m^{2}=0, \tan \beta=30$, $\mu>0$, and $m_{t}=173 \mathrm{GeV}$. Bottom panel: Neutralino-proton spin-independent direct-detection cross-sections $\sigma_{\tilde{\chi}^{0}}^{S I}$ vs. higgsino fraction $f_{\text {Higgsino }}$ for $\Delta m^{2}=0, \tan \beta=30$ and $\mu>0$.

feature reduced electroweak fine-tuning relative to the local parameter space with similar values of $m_{0}$. The amount of fine-tuning with respect to the electroweak scale (EWFT) is typically quantified by the minimal electroweak fine-tuning parameter

$$
\Delta_{E W} \equiv \max \left(C_{i}\right) /\left(M_{Z}^{2} / 2\right)
$$

where $C_{\mu} \equiv\left|-\mu^{2}\right|, C_{H_{u}} \equiv\left|-m_{H_{u}}^{2} \tan ^{2} \beta /\left(\tan ^{2} \beta-1\right)\right|$, and $C_{H_{d}} \equiv\left|-m_{H_{d}}^{2} /\left(\tan ^{2} \beta-1\right)\right|$. The percent-level of EWFT is then given by $\Delta_{E W}^{-1}$. It should be noted that for most of the parameter space explored in this analysis $C_{\mu}$ is dominant, and so generally we have $\Delta_{E W}=\left|-\mu^{2}\right| /\left(M_{Z}^{2} / 2\right)$. In the following, we shall use this parameter to analyze the minimal amount of fine-tuning required regions of the studied parameter space. 


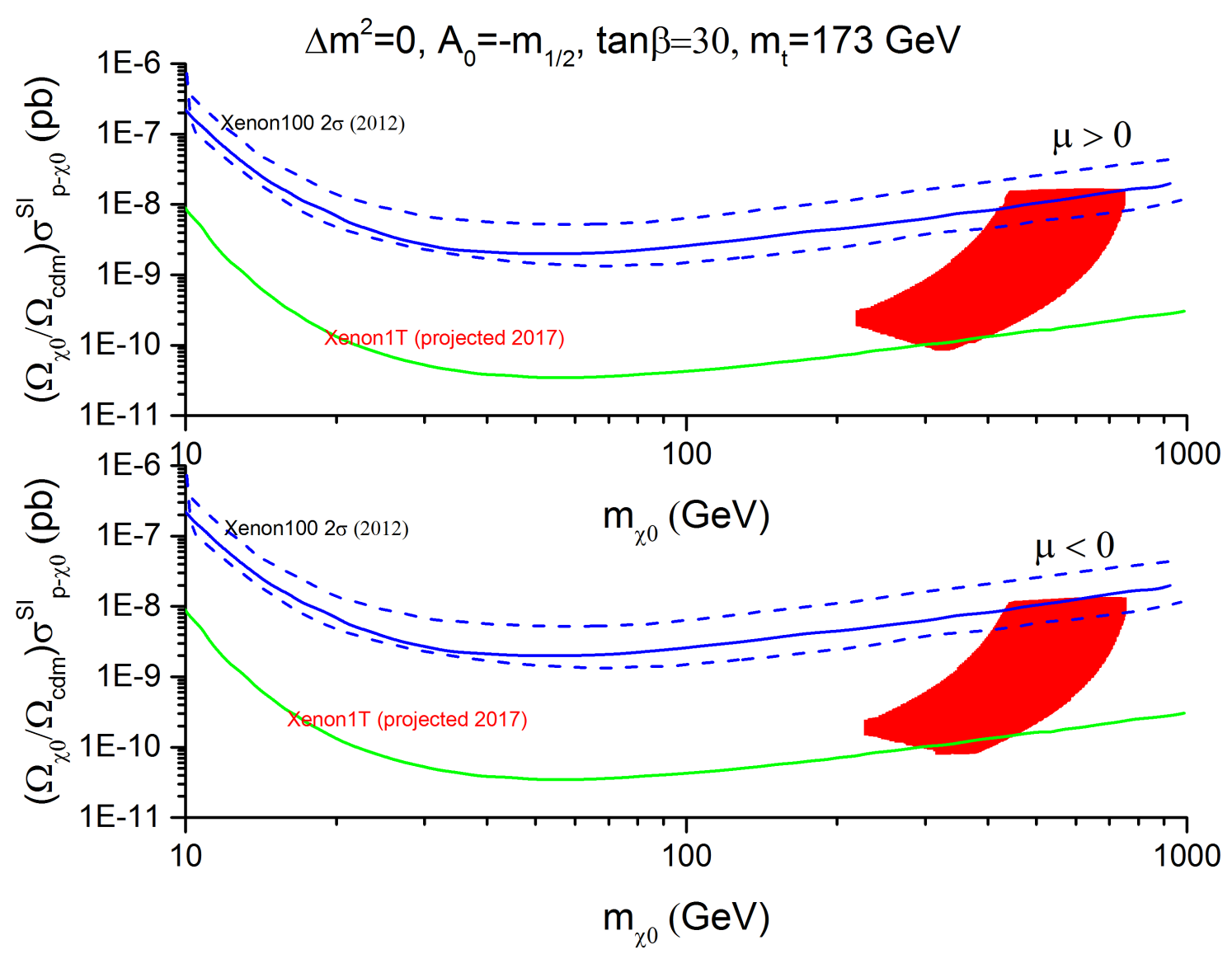

FIG. 10: Re-scaled neutralino spin-independent direct-detection cross-sections $\left(\Omega_{\tilde{\chi}^{0}} / \Omega_{D M}\right) \times \sigma_{\tilde{\chi}^{0}}^{S I}$ vs. LSP mass $m_{\tilde{\chi}^{0}}$ for $\Delta m^{2}=0, \tan \beta=30$, and $m_{t}=173 \mathrm{GeV}$. Here, we take $\Omega_{D M} h^{2}=0.11$. The top panel is for $m u>0$ while the bottom panel shows $m<0$.

In [63], it is argued that $\Delta_{E W}$ only provides a measure of the minimum amount of finetuning in regards to the electroweak scale and provides no information about the high scale physics involved in a particular model of SUSY breaking. In order to provide a measure of how fine-tuned a particular model is given knowledge of how SUSY is broken at a high energy scale, a parameter called $\Delta_{H S}$ was introduced which is analogous to $\Delta_{E W}[63$. For most of the parameter space this parameter is given by

$$
\Delta_{H S}=\frac{m_{0}^{2}+\mu^{2}}{\left(M_{Z}^{2} / 2\right)}=\Delta_{E W}+\frac{m_{0}^{2}}{\left(M_{Z}^{2} / 2\right)} .
$$

As we can see, for regions of the mSUGRA/CMSSM parameter space with large scalar masses, $\Delta_{H S}$ is very large even for those cases where $\Delta_{E W}$ is small such as in the HB regions. This simply reflects the fact that, although a particular SUSY spectrum may be completely 


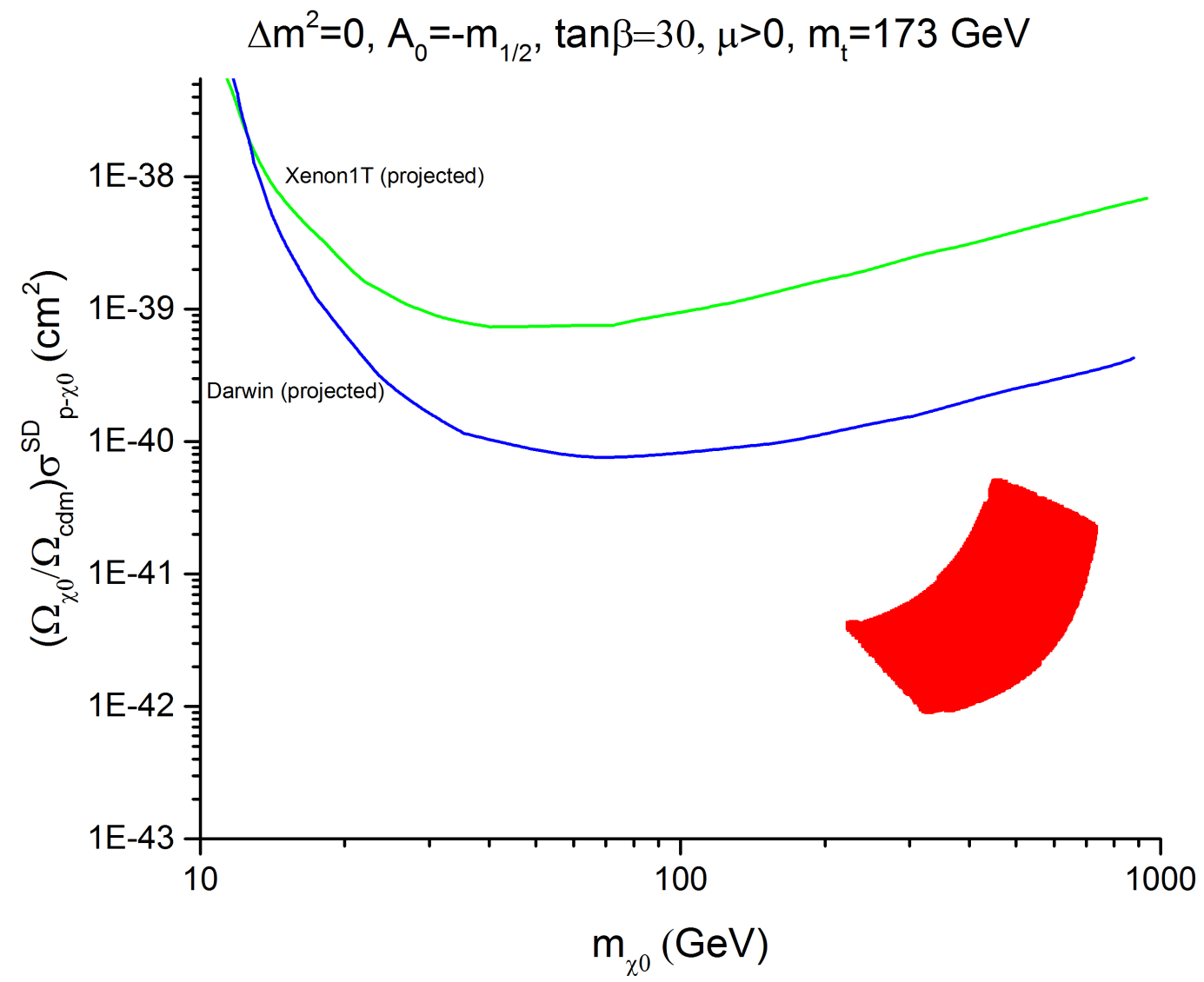

FIG. 11: Re-scaled neutralino spin-dependent direct-detection cross-sections $\left(\Omega_{\tilde{\chi}^{0}} / \Omega_{D M}\right) \times \sigma_{\tilde{\chi}^{0}}^{S D}$ vs. LSP mass $m_{\tilde{\chi}^{0}}$ for $\Delta m^{2}=0, \tan \beta=30, \mu>0$, and $m_{t}=173 \mathrm{GeV}$. Here, we take $\Omega_{D M} h^{2}=0.11$. Also shown are the projected reach for the Xenon1T [109] and Darwin [110] dark matter directdetection experiments.

natural and solve the hierarchy problem without any fine-tuning, obtaining this spectrum within the mSUGRA/CMSSM framework of SUSY breaking requires large cancellations which only happens for specific sets of soft-terms rather than the general parameter space. For the specific string model at hand, we will assume that high-scale parameters $m_{3 / 2}$ and $\theta$, and thus $m_{1 / 2}$ and $m_{0}$, are chosen by whatever dynamics are responsible for moduli stabilization, thus at least providing an explanation for the choice of high-scale parameters. We leave this topic for future work.

Contour plots of the $m_{1 / 2}$ vs. $m_{H}=m_{0}$ plane with $\Delta m^{2}=0$ for $\tan \beta=30$ are shown for $\mu>0$ and $\mu<0$ respectively in Figs. 2,3. The mass of the lightest CP-even Higgs is between 


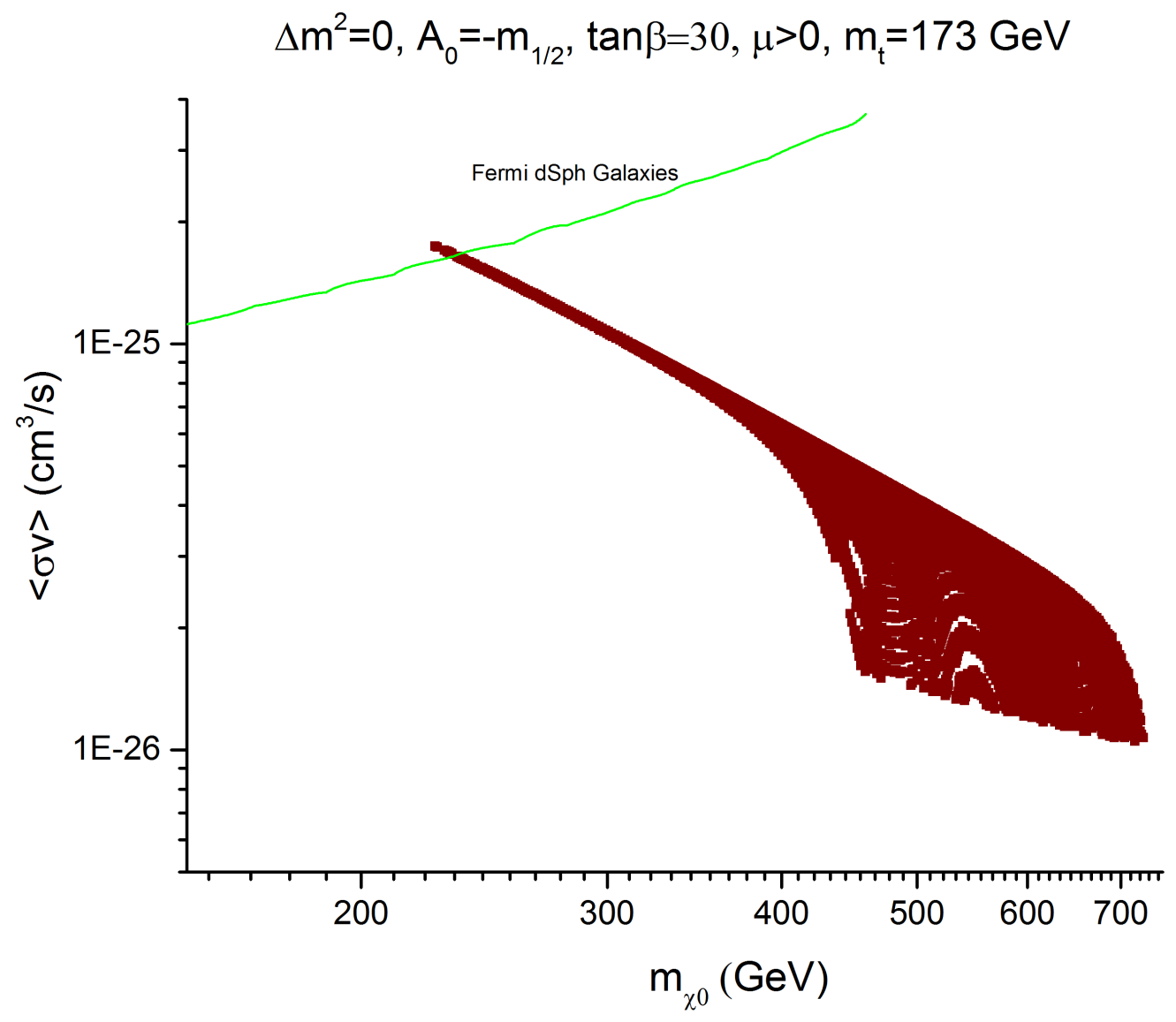

FIG. 12: Annihilation cross-sections to continuum photons $<\sigma v>$ vs. LSP neutralino mass $m_{\tilde{\chi}_{0}}$ for model points satisfying $\Omega_{\chi_{0}} h^{2} \lesssim 0.123$ for $\Delta m^{2}=0, \tan \beta=30, \mu>0$, and $m_{t}=173 \mathrm{GeV}$.

$124-126 \mathrm{GeV}$ for the regions shown. The viable areas of the parameter space lie along the $\mathrm{HB} / \mathrm{FP}$ regions of the parameter space where $m_{0}=m_{H} \sim(4-5) \times m_{1 / 2}$. As can be seen from Fig. ??, these regions can be obtained from the intersecting/magnetized D-brane model for $\theta \approx-(0.6-0.7) \mathrm{rad}$. The dark blue regions in the figures have a relic density which satisfies $\Omega_{\tilde{\chi_{0}}} h^{2}<=0.0123$, the light blue regions satisfy $0.015<=\Omega_{\tilde{\chi_{0}}} h^{2}<=0.05$, while the regions in green satisfy $0.105<=\Omega_{\tilde{\chi_{0}}} h^{2}<=0.123$ corresponding to the 9 -year $2 \sigma$ bounds on the dark matter density observed by WMAP. The gray regions have a relic density larger than the WMAP and Planck bounds. The upper panel of the plot shows values of the Higgs mass indicated by solid black contours and are in the range $124-126 \mathrm{GeV}$, while values of the electroweak fine-tuning parameter $\Delta_{E W}$ are indicated by dashed orange contours. For the parameter space with $\Omega_{\tilde{\chi_{0}}} h^{2}<=0.0123, \Delta_{E W} \lesssim 130$. The bottom panel of the plot 
TABLE I: Benchmark points for for $\Delta m^{2}=0, \tan \beta=30, \mu>0, m_{t}=173 \mathrm{GeV}$, and $\Delta_{E W} \lesssim 30$.

\begin{tabular}{|c|c|c|c|c|c|c|c|c|}
\hline$m_{0}(\mathrm{GeV})$ & $m_{1 / 2}(\mathrm{GeV})$ & $m_{h}(\mathrm{GeV})$ & $m_{\tilde{\chi}^{0}}(\mathrm{GeV})$ & $m_{\tilde{g}}(\mathrm{TeV})$ & $m_{\tilde{t}_{1}}(\mathrm{TeV})$ & $\Omega_{\chi_{0}} h^{2}$ & $\sigma_{\tilde{\chi}_{0}}^{S I}(\mathrm{pb})$ & $\Delta_{E W}$ \\
\hline \hline 5200 & 1059.0 & 124.0 & 236.4 & 2.58 & 3.48 & $8.6 \times 10^{-3}$ & $3.2 \times 10^{-9}$ & 13.7 \\
5500 & 1153.5 & 124.3 & 253.3 & 2.79 & 3.71 & $9.3 \times 10^{-3}$ & $2.6 \times 10^{-9}$ & 15.6 \\
5750 & 1233.0 & 124.5 & 263.5 & 2.96 & 3.90 & $9.7 \times 10^{-3}$ & $2.2 \times 10^{-9}$ & 16.7 \\
6000 & 1313.5 & 124.7 & 276.4 & 3.13 & 4.09 & $1.0 \times 10^{-2}$ & $1.9 \times 10^{-9}$ & 18.3 \\
6250 & 1397.3 & 124.9 & 294.5 & 3.31 & 4.28 & $1.1 \times 10^{-2}$ & $1.7 \times 10^{-9}$ & 20.6 \\
6500 & 1479.0 & 125.1 & 301.4 & 3.49 & 4.47 & $1.2 \times 10^{-2}$ & $1.4 \times 10^{-9}$ & 21.9 \\
6750 & 1562.0 & 125.3 & 311.1 & 3.67 & 4.66 & $1.2 \times 10^{-2}$ & $1.2 \times 10^{-9}$ & 22.8 \\
7000 & 1646.5 & 125.5 & 320.5 & 3.85 & 4.85 & $1.3 \times 10^{-2}$ & $1.1 \times 10^{-9}$ & 24.1 \\
7250 & 1732.0 & 125.7 & 332.5 & 4.03 & 5.05 & $1.3 \times 10^{-2}$ & $9.7 \times 10^{-10}$ & 25.9 \\
7500 & 1818.0 & 125.9 & 342.5 & 4.21 & 5.24 & $1.4 \times 10^{-2}$ & $8.6 \times 10^{-10}$ & 27.3 \\
7750 & 1905.0 & 126.0 & 350.1 & 4.39 & 5.44 & $1.5 \times 10^{-2}$ & $7.6 \times 10^{-10}$ & 28.5 \\
8000 & 1992.5 & 126.2 & 358.2 & 4.57 & 5.63 & $1.5 \times 10^{-2}$ & $6.7 \times 10^{-10}$ & 29.8 \\
\hline
\end{tabular}

indicates values for the Flavor Changing Neutral Current (FCNC) process, $b \rightarrow s \gamma$ by solid black contours and are in the range $(3.27-3.30) \times 10^{-4}$, while dashed magenta contours indicate values for the process $B_{s}^{0} \rightarrow \mu^{+} \mu^{-}$and are in the range $(3.03-3.04) \times 10^{-9}$ consistent with experimental constraints. As can be observed, the parameter spaces for both signs of $\mu$ are very similar. In addition, it should be noted that the parameter space for different values of $\tan \beta$ are also very similar, although here we have only shown the parameter space for $\tan \beta=30$. In addition, varying the top quark mass within its experimental uncertainty shifts the parameter space slightly in the $m_{1 / 2}$ vs. $m_{H}=m_{0}$ plane, however essentially the same results are obtained. Please note that although these plots seem to indicate that these spectra lie along a continuous band, they are actually interspersed with spectra where SuSpect is not able to converge to a solution.

Plots of the parameter space with $\Delta m^{2}=0.1 m_{H}^{2}$ are shown in Figs. 4 and 5 for $\tan \beta=30$ with $\mu>0$ and $\mu<0$ respectively, while plots of the parameter space with $\Delta m^{2}=-0.1 m_{H}^{2}$ are shown in Figs. 6 and 7 for $\tan \beta=30$ with $\mu>0$ and $\mu<0$ respectively. As we can see from these plots, the HB/FP regions are still present even when the sfermion mass-squared 
TABLE II: Benchmark points for for $\Delta m^{2}=0, \tan \beta=30, \mu>0, m_{t}=173.3 \mathrm{GeV}$, and $0.105 \leq$ $\Omega_{\chi_{0}} h^{2} \geq 0.123$.

\begin{tabular}{|c|c|c|c|c|c|c|c|c|}
\hline$m_{0}(\mathrm{GeV})$ & $m_{1 / 2}(\mathrm{GeV})$ & $m_{h}(\mathrm{GeV})$ & $m_{\tilde{\chi}^{0}}(\mathrm{GeV})$ & $m_{\tilde{g}}(\mathrm{TeV})$ & $m_{\tilde{t}_{1}}(\mathrm{TeV})$ & $\Omega_{\chi_{0}} h^{2}$ & $\sigma_{\tilde{\chi}_{0}}^{S I}(\mathrm{pb})$ & $\Delta_{E W}$ \\
\hline \hline 5000 & 1082.0 & 123.7 & 454.7 & 2.26 & 3.39 & $1.1 \times 10^{-1}$ & $1.3 \times 10^{-8}$ & 58.1 \\
5190 & 1146.0 & 123.9 & 483.3 & 2.76 & 3.54 & $1.1 \times 10^{-1}$ & $1.3 \times 10^{-8}$ & 64.6 \\
5510 & 1257.0 & 124.3 & 533.7 & 3.00 & 3.79 & $1.2 \times 10^{-1}$ & $1.3 \times 10^{-8}$ & 77.0 \\
5740 & 1336.0 & 124.5 & 567.8 & 3.17 & 3.97 & $1.2 \times 10^{-1}$ & $1.3 \times 10^{-8}$ & 85.5 \\
6000 & 1427.0 & 124.7 & 607.5 & 3.36 & 4.17 & $1.1 \times 10^{-1}$ & $1.3 \times 10^{-8}$ & 96.1 \\
6250 & 1518.0 & 125.0 & 649.0 & 3.56 & 4.37 & $1.2 \times 10^{-1}$ & $1.3 \times 10^{-8}$ & 108.6 \\
6500 & 1606.0 & 125.2 & 685.2 & 3.74 & 4.56 & $1.1 \times 10^{-1}$ & $1.3 \times 10^{-8}$ & 118.9 \\
6750 & 1697.0 & 125.4 & 723.6 & 3.93 & 4.76 & $1.1 \times 10^{-1}$ & $1.3 \times 10^{-8}$ & 130.9 \\
\hline
\end{tabular}

terms are split about the Higgs scalar mass-squared term. Similar results are obtained for varying levels of splitting. This is, of course, expected since it was previously observed that any boundary conditions of the form $\left(m_{H_{u}}^{2}, m_{U_{3}}^{2}, m_{Q_{3}}^{2}\right) \propto(1,1+x, 1-x)$ with $x$ an arbitrary constant may give rise to focus points. In the D-brane model at hand, the amount of splitting depends upon the Kähler moduli as well as on the Goldstino angle $\theta$. These results demonstrate that the $\mathrm{HB} / \mathrm{FP}$ region is present for arbitrary values of the Kähler moduli.

A plot of the relic density vs. LSP mass is shown in the top-right panel of Fig. 8 for $\Delta m^{2}=0, \tan \beta=30$, and $\mu>0$. Here, we can see that the LSP mass is in the range $\approx 450-750 \mathrm{GeV}$ for superpartner spectra where the relic density falls within the WMAP $2 \sigma$ bounds, $0.105<=\Omega_{\tilde{\chi_{0}}} h^{2}<=0.123$. Also from this plot, we can see that when the LSP mass is in the range $240-350 \mathrm{GeV}$, the relic density is minimum, $\Omega_{\tilde{\chi}_{0}} h^{2} \approx 0.010-0.015$. A plot of the higgsino fraction which composes the LSP vs. the LSP mass is shown in the upper-left panel of Fig. 8. From this plot, it can be seen that for LSP's with masses in the range $240-350 \mathrm{GeV}$ are almost pure higgsino.

For a scenario with a mostly higgsino LSP which composes roughly $10 \%$ of the observed dark matter density, it has been suggested that the bulk of the dark matter is composed of axions [103]. In the present context, superheavy hidden sector states may also provide 
some component of the dark matter. Such states tend to generically carry fractional electric charges, however they may be confined into neutral bound states by the hidden sector gauge interaction [104], similar to how quarks become bound into hadrons. It is known that superheavy particles $X$ with masses in the range $10^{11} \lesssim M_{X} \lesssim 10^{14}$ might well have been produced naturally through the interaction of the vacuum with the gravitational field during the reheating period of the Universe following inflation in numbers sufficient to produce superheavy dark matter [105]. For the model under study, it was shown that the hidden sector states become confined at high energy scales $10^{11}-10^{12} \mathrm{GeV}$ [25], just in the preferred range. Thus, these bound states are a natural candidate for dark matter, in addition to the neutralino LSP.

In the upper panel of Fig. 9, a strong correlation between the relic density and the higgsino fraction $f_{\text {higgsino }}$ of the linear combination of states composing the LSP may be observed. It can be seen from this plot that the miminal relic density occurs for an almost pure higgsino LSP. It may be inferred from this that the higher the higgsino fraction composing the LSP, the lighter the LSP mass. This result is, of course, not unexpected. For spectra satisfying $0.105<=\Omega_{\tilde{\chi_{0}}} h^{2}<=0.123$, the higgsino fraction is in the range $0.65-0.85$ with the rest being primarily of bino composition. In general, the higher the bino fraction, the larger the relic density and the larger the LSP mass. In addition, there is a vanishingly small wino fraction.

The lower-left panel of Fig. 8 shows a plot of $\mu$ vs. LSP mass, from which is can be seen that $m_{\tilde{\chi_{0}}} \approx \mu$. The lower-right panel of Fig. 8 displays a plot of the minimal electroweak finetuning parameter $\Delta_{E W}$ vs. LSP mass. From this plot it can be seen that $\Delta_{E W} \sim(50-120)$ for superpartner spectra where $0.105<=\Omega_{\tilde{\chi}_{0}} h^{2}<=0.123$. Thus, such spectra are minimally fine-tuned at the level of $\Delta_{E W}^{-1}=1-2 \%$. For those spectra with a light higgsino-like LSP with a mass in the range $230-350 \mathrm{GeV}$, the minimal fine-tuning is much less, in the range of $\Delta_{E W}^{-1}=3-7 \%$. This result can generally be understood given that the $\mu$-parameter is essentially equivalent to the higgsino mass as well as being a measure of the minimal electreak fine-tuning, viz-á-viz $C_{\mu} \equiv\left|-\mu^{2}\right|$.

For all of the spectra for which the Higgs mass satisfies $124 \mathrm{GeV} \lesssim m_{h} \lesssim 126 \mathrm{GeV}$ and for which the relic density satisfies the WMAP and Planck constraints, the gluino mass is in the range $2.6-4.4 \mathrm{TeV}$, while the lightest stop mass is in the range $3.5-5.6 \mathrm{TeV}$. The squarks and sleptons all have masses greater than the gluino mass. Sets of benchmark points 
are shown in Table $\mathrm{I}$ and Table $\mathrm{II}$ spanning a range of Higgs masses from $124-126 \mathrm{GeV}$ for the cases $\Delta_{E W} \lesssim 30$ and $0.105 \leq \Omega_{\chi_{0}} h^{2} \geq 0.123$ respectively. In particular, only the four lightest neutralinos and charginos have masses which are below $1 \mathrm{TeV}$, with a small mass splitting of $5-10 \mathrm{GeV}$ between the LSP and NLSP. These spectra are very similar to those obtained in RNS models with a light higgsino LSP. The possible signals that may be observed for these spectra have been considered in [68]. Due to the heavy masses for the gluino and squarks in these models, it would be very difficult to observe a clear and distinct signal at the LHC if the spectrum of superpartners falls into these regions of the parameter space. Direct production of light higgsinos may produce clear, soft trilepton signatures if the mass gap between the LSP and NLSP is not too great. The prospects for observing superpartners at a linear collider or at a higher-energy hadron collider appear to be somewhat more promising [106]. In the case of the linear collider, this is because lightest neutralinos and charginos may be produced even with a lower CM energy and their decays may be studied with cleaner backgrounds, whereas it may be possible to directly produce stops and gluinos at a hadron collider with higher collision energy.

Although it may be difficult to observe a distinct signal at the LHC if nature has chosen one of these spectra, the situation is somewhat better in the case of direct-detection experiments such as the upcoming XENON1-T [71] and superCDMS [72] experiments which will probe the spin-indepedent cross-sections for WIMP dark matter. The spin-independent direct-detection cross-sections are plotted vs. higgsino fraction for the neutralino LSP in the lower panel of Fig. 9. Here, it can be clearly seen that the cross-sections become much smaller with a larger higgsino fraction. This can be easily understood as being due to the weakness with which higgsinos couple. In Fig. 10, the re-scaled spin-independent crosssections are plotted vs. LSP mass, where the cross-sections have been re-scaled by the ratio of the neutralino relic density to the upper limit on the 9-year $2 \sigma$ WMAP bound, $\Omega_{D M} h^{2}<=0.123$. This has the effect of lowering the effective cross-section on the pure higgsino LSP by roughly a factor of ten since the relic density in this case is roughly a factor of ten lower than the dark matter density observed by WMAP and Planck. Also shown on the Figure is the XENON100 upper limit on the SI proton-neutralino cross-section [107, 108] as well as the projected reach of the next generation XENON1-T experiment [71]. In the Figure, it can be seen that spectra with a higher LSP mass are ruled out or nearly ruled out by the most recent XENON100 results. Other points in the parameter space should be 
completely covered by the upcoming XENON1-T experiment including those with a light higgsino LSP, which are just within reach. However, the spin-dependent direct-detection cross-sections may not be in reach as shown in Fig. 11, [109, 110].

Also of great interest are the prospects for indirect dark matter detection resulting from neutralino dark matter annihilations. The annihilation cross sections to continuum photons are shown in Fig. 12 for model points satisfying $\Omega_{D M} h^{2}<=0.123$ with $\Delta m^{2}=0, \tan \beta=30$, $\mu>0$, and $m_{t}=173 \mathrm{GeV}$. For comparison, the constraints derived from a combined analysis of dwarf spheroidal (dSph) Milky Way galaxies [111,112] are also shown. As can be seen from this plot most of the model points, specifically those with a neutralino mass $m_{\tilde{\chi}_{0}} \gtrsim 250 \mathrm{GeV}$, are below the Fermi dSph constraint.

\section{CONCLUSION}

When supersymmetry breaking is dominated by the complex structure moduli and the universal dilaton, a subset of the supersymmetry parameter space in a semi-realistic MSSM constructed from intersecting/magnetized D-branes is similar to the mSUGRA/CMSSM parameter space with the trilinear term fixed to be minus the gaugino mass, $A_{0}=-m_{1 / 2}$.

More generally, the scalar mass-squared terms for sfermions are split about the Higgs masssquared terms, $m_{Q_{L}, L_{L}}^{2}=m_{H}^{2}-\Delta m^{2}$ and $m_{Q_{R}, L_{R}}^{2}=m_{H}^{2}+\Delta m^{2}$, for generic values of the Kähler moduli. The hyberbolic branch/focus point (HB/FP) regions of this parameter space are present for both $\Delta m^{2}=0$ and $\Delta m^{2} \neq 0$. In this work, we have studied these regions in detail.

In the case of the high-scale boundary conditions obtained from the model for supersupersymmetry breaking dominated by the complex structure moduli and the dilaton, it is very interesting that they take the form $\left(m_{H_{u}}^{2}, m_{U_{3}}^{2}, m_{Q_{3}}^{2}\right) \propto(1,1+x, 1-x)$ with $x$ an arbitrary constant. In the present context, $\mathrm{x}$ is a function of the Kähler moduli in context of this model. As was discussed, it has been known for some time that focus points may be obtained for any boundary conditions of this form. Thus, the HB/FP region discussed for $\Delta m^{2}=x=0$ remains present even when $\Delta m^{2}=x \neq 0$. Our results verified that this was indeed the case. With respect to the intersecting/magnetized D-brane model, this implies that the resulting physics does not depend upon the choice of Kähler moduli.

It has been shown that there exists superpartner spectra with a light Higgsino-like LSP 
with a mass in the range $230-350 \mathrm{GeV}$ and a Higgs mass in the range $124-126 \mathrm{GeV}$, and which satisfy most standard experimental constraints. Consequently, viable spectra with low EWFT between $3-7 \%$ may be obtained. The rescaled spin-independent direct-detection crosssections are in range of future experiments such as XENON-1T and super CDMS, while the relic density is smaller than the WMAP and Planck bounds by roughly a factor of ten. For superpartner spectra which fall within the WMAP 9-year $2 \sigma$ bounds, the LSP mass is in the range $450-750 \mathrm{GeV}$. These spectra require a minimum fine-tuning of $1-2 \%$. In addition, a portion of this parameter space is already excluded by the XENON100 results. Thus, the parameter space with a nearly pure higgsino composition is favored. However this implies that the LSP provides $\sim 10 \%$ of the dark matter density observed by WMAP and Planck. Axions or superheavy hidden-sector dark matter may then provide the bulk of the astrophysical dark matter. Also of great interest are the prospects for indirect dark matter detection resulting from neutralino dark matter annihilations. The annihilation cross sections to continuum photons for most of the model points, specifically those with a neutralino mass $m_{\tilde{\chi}_{0}} \gtrsim 250 \mathrm{GeV}$, are below the Fermi dSph constraint.

For all of these spectra, the gluino mass and the scalar masses are all in the multi-TeV range. In particular, the gluino mass is in the range $2.6-4.4 \mathrm{TeV}$ while the lightest stop mass is in the range $3.5-5.6 \mathrm{TeV}$. The other squark and slepton masses are typically in the $5-10 \mathrm{TeV}$ range, as are the masses of the additional Higgs scalars. Due to the heavy masses for the gluino and squarks in these models, it would be very difficult to observe a clear and distinct signal at the LHC if the spectrum of superpartners falls into these regions of the parameter space. However, the prospects for observing superpartners at a linear collider or at a higher-energy hadron collider appear to be more promising. We shall soon see as LHC run II is currently underway at a higher CM energy of $13 \mathrm{TeV}$.

\section{ACKNOWLEDGMENTS}

VEM would like to thank the University of Houston-Clear Lake Natural Sciences division for the purchase of a high-performance Linux workstation which was used to complete this work. 
[1] W. Buchmuller, K. Hamaguchi, O. Lebedev and M. Ratz, Phys. Rev. Lett. 96, 121602 (2006), and references therein.

[2] O. Lebedev, H. P. Nilles, S. Raby, S. Ramos-Sanchez, M. Ratz, P. K. S. Vaudrevange and A. Wingerter, Phys. Lett. B 645, 88 (2007), and references therein.

[3] J. E. Kim and B. Kyae, Nucl. Phys. B 770, 47 (2007); Phys. Rev. D 77, 106008 (2008); J. H. Huh, J. E. Kim and B. Kyae, arXiv:0904.1108 [hep-ph].

[4] V. Braun, Y. H. He, B. A. Ovrut and T. Pantev, Phys. Lett. B 618, 252 (2005); JHEP 0605, 043 (2006), and references therein.

[5] V. Bouchard and R. Donagi, Phys. Lett. B 633, 783 (2006), and references therein.

[6] I. Antoniadis, J. R. Ellis, J. S. Hagelin and D. V. Nanopoulos, Phys. Lett. B 205 (1988) 459; Phys. Lett. B 208 (1988) 209 [Addendum-ibid. B 213 (1988) 562]; Phys. Lett. B 231 (1989) 65.

[7] A. E. Faraggi, D. V. Nanopoulos and K. J. Yuan, Nucl. Phys. B 335, 347 (1990).

[8] I. Antoniadis, G. K. Leontaris and J. Rizos, Phys. Lett. B 245, 161 (1990).

[9] J. L. Lopez, D. V. Nanopoulos and K. J. Yuan, Nucl. Phys. B 399, 654 (1993); D. V. Nanopoulos, hep-ph/0211128.

[10] G. B. Cleaver, A. E. Faraggi, D. V. Nanopoulos and J. W. Walker, Nucl. Phys. B 620, 259 (2002), and references therein.

[11] M. Berkooz, M. R. Douglas and R. G. Leigh, Nucl. Phys. B 480, 265 (1996).

[12] L. E. Ibanez, F. Marchesano and R. Rabadan, JHEP 0111, 002 (2001).

[13] R. Blumenhagen, B. Kors, D. Lust and T. Ott, Nucl. Phys. B 616, 3 (2001).

[14] M. Cvetič, G. Shiu and A. M. Uranga, Phys. Rev. Lett. 87, 201801 (2001); M. Cvetič, G. Shiu and A. M. Uranga, Nucl. Phys. B 615, 3 (2001).

[15] M. Cvetič, I. Papadimitriou and G. Shiu, Nucl. Phys. B 659, 193 (2003) [Erratum-ibid. B 696, 298 (2004)].

[16] M. Cvetic, T. Li and T. Liu, Nucl. Phys. B 698, 163 (2004) hep-th/0403061.

[17] M. Cvetic, P. Langacker, T. Li and T. Liu, Nucl. Phys. B 709, 241 (2005) hep-th/0407178.

[18] M. Cvetic, T. Li and T. Liu, Phys. Rev. D 71, 106008 (2005) hep-th/0501041. 
[19] C.-M. Chen, G. V. Kraniotis, V. E. Mayes, D. V. Nanopoulos and J. W. Walker, Phys. Lett. B 611, 156 (2005); Phys. Lett. B 625, 96 (2005).

[20] C. M. Chen, T. Li and D. V. Nanopoulos, Nucl. Phys. B 732, 224 (2006).

[21] R. Blumenhagen, M. Cvetic, P. Langacker and G. Shiu, Ann. Rev. Nucl. Part. Sci. 55, 71 (2005), and references therein.

[22] T. P. T. Dijkstra, L. R. Huiszoon and A. N. Schellekens, Phys. Lett. B 609, 408 (2005).

[23] T. P. T. Dijkstra, L. R. Huiszoon and A. N. Schellekens, Nucl. Phys. B 710, 3 (2005), and references therein.

[24] V. E. Mayes, Nucl. Phys. B 877, 401 (2013) arXiv:1305.2842 [hep-ph]].

[25] C. -M. Chen, T. Li, V. E. Mayes and D. V. Nanopoulos, Phys. Rev. D 77, 125023 (2008) arXiv:0711.0396 [hep-ph]].

[26] C. -M. Chen, T. Li, V. E. Mayes and D. V. Nanopoulos, Phys. Lett. B 665, 267 (2008) hep-th/0703280.

[27] M. S. Carena and H. E. Haber, Prog. Part. Nucl. Phys. 50, 63 (2003) hep-ph/0208209.

[28] J. R. Ellis, J. S. Hagelin, D. V. Nanopoulos and M. Srednicki, Phys. Lett. B 127, 233 (1983).

[29] J. R. Ellis, J. S. Hagelin, D. V. Nanopoulos, K. A. Olive and M. Srednicki, Nucl. Phys. B 238, 453 (1984).

[30] J. R. Ellis, D. V. Nanopoulos and K. Tamvakis, Phys. Lett. B 121, 123 (1983).

[31] S. Dimopoulos, S. Raby and F. Wilczek, Phys. Rev. D 24, 1681 (1981).

[32] L. E. Ibanez and G. G. Ross, Phys. Lett. B 105, 439 (1981).

[33] G. Aad et al. [ATLAS Collaboration], Phys. Lett. B 716, 1 (2012) arXiv:1207.7214 [hep-ex]].

[34] S. Chatrchyan et al. [CMS Collaboration], Phys. Lett. B 716, 30 (2012) arXiv:1207.7235 [hep-ex]].

[35] G. Aad et al. [ATLAS Collaboration], Phys. Rev. D 87, 012008 (2013) arXiv:1208.0949 [hep-ex]].

[36] G. Aad et al. [ATLAS Collaboration], JHEP 1207, 167 (2012) arXiv:1206.1760 [hep-ex]].

[37] S. Chatrchyan et al. [CMS Collaboration], Phys. Rev. Lett. 109, 171803 (2012) arXiv:1207.1898 [hep-ex]].

[38] G. Aad et al. [ATLAS Collaboration], Phys. Lett. B 710, 67 (2012) arXiv:1109.6572 [hep- 
$\mathrm{ex}]$.

[39] S. Chatrchyan et al. [CMS Collaboration], Phys. Rev. Lett. 107, 221804 (2011) arXiv:1109.2352 [hep-ex]].

[40] A. H. Chamseddine, R. L. Arnowitt and P. Nath, Phys. Rev. Lett. 49, 970 (1982).

[41] N. Ohta, Prog. Theor. Phys. 70, 542 (1983).

[42] L. J. Hall, J. D. Lykken and S. Weinberg, Phys. Rev. D 27, 2359 (1983).

[43] K. L. Chan, U. Chattopadhyay and P. Nath, Phys. Rev. D 58, 096004 (1998) hepph/9710473.

[44] J. L. Feng, K. T. Matchev and T. Moroi, Phys. Rev. Lett. 84, 2322 (2000) hep-ph/9908309.

[45] J. L. Feng, K. T. Matchev and T. Moroi, Phys. Rev. D 61, 075005 (2000) hep-ph/9909334.

[46] H. Baer, C. -h. Chen, F. Paige and X. Tata, Phys. Rev. D 52, 2746 (1995) hep-ph/9503271].

[47] H. Baer, C. -h. Chen, M. Drees, F. Paige and X. Tata, Phys. Rev. D 59, 055014 (1999) hep-ph/9809223.

[48] U. Chattopadhyay, A. Corsetti and P. Nath, Phys. Rev. D 68, 035005 (2003) hep$\mathrm{ph} / 0303201$.

[49] M. Kadastik, K. Kannike, A. Racioppi and M. Raidal, JHEP 1205, 061 (2012) arXiv:1112.3647 [hep-ph]].

[50] C. Strege, G. Bertone, D. G. Cerdeno, M. Fornasa, R. Ruiz de Austri and R. Trotta, JCAP 1203, 030 (2012) arXiv:1112.4192 [hep-ph]].

[51] L. Aparicio, D. G. Cerdeno and L. E. Ibanez, JHEP 1204, 126 (2012) arXiv:1202.0822 [hep-ph]].

[52] J. Ellis and K. A. Olive, Eur. Phys. J. C 72, 2005 (2012) arXiv:1202.3262 [hep-ph]].

[53] H. Baer, V. Barger and A. Mustafayev, JHEP 1205, 091 (2012) arXiv:1202.4038 [hep-ph]].

[54] K. Matchev and R. Remington, arXiv:1202.6580 [hep-ph].

[55] S. Akula, P. Nath and G. Peim, Phys. Lett. B 717, 188 (2012) arXiv:1207.1839 [hep-ph]].

[56] D. Ghosh, M. Guchait, S. Raychaudhuri and D. Sengupta, Phys. Rev. D 86, 055007 (2012) arXiv:1205.2283 [hep-ph]].

[57] A. Fowlie, M. Kazana, K. Kowalska, S. Munir, L. Roszkowski, E. M. Sessolo, S. Trojanowski and Y. -L. S. Tsai, Phys. Rev. D 86, 075010 (2012) arXiv:1206.0264 [hep-ph]]. 
[58] O. Buchmueller, R. Cavanaugh, M. Citron, A. De Roeck, M. J. Dolan, J. R. Ellis, H. Flacher and S. Heinemeyer et al., Eur. Phys. J. C 72, 2243 (2012) [arXiv:1207.7315 [hep-ph]].

[59] C. Strege, G. Bertone, F. Feroz, M. Fornasa, R. Ruiz de Austri and R. Trotta, JCAP 1304, 013 (2013) arXiv:1212.2636 [hep-ph]].

[60] M. Citron, J. Ellis, F. Luo, J. Marrouche, K. A. Olive and K. J. de Vries, Phys. Rev. D 87, 036012 (2013) arXiv:1212.2886 [hep-ph]].

[61] J. Ellis, F. Luo, K. A. Olive and P. Sandick, Eur. Phys. J. C 73, no. 4, 2403 (2013) arXiv:1212.4476 [hep-ph]].

[62] C. Boehm, J. Da Silva, A. Mazumdar and E. Pukartas, Phys. Rev. D 87, 023529 (2013) arXiv:1205.2815 [hep-ph]].

[63] H. Baer, V. Barger, P. Huang, D. Mickelson, A. Mustafayev and X. Tata, Phys. Rev. D 87, no. 3, 035017 (2013) arXiv:1210.3019 [hep-ph]].

[64] J. R. Ellis, K. A. Olive and Y. Santoso, Phys. Lett. B 539, 107 (2002) hep-ph/0204192.

[65] J. R. Ellis, T. Falk, K. A. Olive and Y. Santoso, Nucl. Phys. B 652, 259 (2003) hep$\mathrm{ph} / 0210205$.

[66] H. Baer, A. Mustafayev, S. Profumo, A. Belyaev and X. Tata, Phys. Rev. D 71, 095008 (2005) [hep-ph/0412059].

[67] H. Baer, A. Mustafayev, S. Profumo, A. Belyaev and X. Tata, JHEP 0507, 065 (2005) hep-ph/0504001.

[68] H. Baer, V. Barger, P. Huang, D. Mickelson, A. Mustafayev, W. Sreethawong and X. Tata, JHEP 1312, 013 (2013) arXiv:1310.4858 [hep-ph]]

[69] J. A. Maxin, V. E. Mayes and D. V. Nanopoulos, Phys. Rev. D 81, 015008 (2010) arXiv:0908.0915 [hep-ph]].

[70] T. Li, D. V. Nanopoulos, S. Raza and X. C. Wang, JHEP 1408, 128 (2014) arXiv:1406.5574 [hep-ph]].

[71] E. Aprile [XENON1T Collaboration], Springer Proc. Phys. 148, 93 (2013) arXiv:1206.6288 [astro-ph.IM]].

[72] D. S. Akerib, M. J. Attisha, C. N. Bailey, L. Baudis, D. A. Bauer, P. L. Brink, P. P. Brusov and R. Bunker et al., Nucl. Instrum. Meth. A 559, 411 (2006).

[73] J. A. Maxin, V. E. Mayes and D. V. Nanopoulos, Phys. Rev. D 84, 106009 (2011) 
arXiv:1108.0887 [hep-ph]].

[74] V. E. Mayes, Int. J. Mod. Phys. A 28, 1350061 (2013) arXiv:1302.4394 [hep-ph]].

[75] M. Drees, R. Godbole and P. Roy, Hackensack, USA: World Scientific (2004) 555 p

[76] R. Blumenhagen, B. Kors, D. Lust and S. Stieberger, Phys. Rept. 445, 1 (2007) arXiv:hepth/0610327.

[77] D. Cremades, L. E. Ibáñez and F. Marchesano, JHEP 0207, 009 (2002) arXiv:hepth/0201205.

[78] G. Shiu and S. H. H. Tye, Phys. Rev. D 58, 106007 (1998) arXiv:hep-th/9805157.

[79] M. Cvetič, P. Langacker and G. Shiu, Phys. Rev. D 66, 066004 (2002); Nucl. Phys. B 642, 139 (2002).

[80] D. Lüst and S. Stieberger, arXiv:hep-th/0302221.

[81] I. Antoniadis, E. Kiritsis and T. N. Tomaras, Phys. Lett. B 486, 186 (2000) arXiv:hepph/0004214; R. Blumenhagen, D. Lust and S. Stieberger, JHEP 0307, 036 (2003) arXiv:hep-th/0305146].

[82] D. Cremades, L. E. Ibáñez and F. Marchesano, JHEP 0307, 038 (2003) arXiv:hepth/0302105.

[83] M. Cvetič and I. Papadimitriou, Phys. Rev. D 68, 046001 (2003) [Erratum-ibid. D 70, 029903 (2004)] arXiv:hep-th/0303083.

[84] B. Körs and P. Nath, Nucl. Phys. B 681, 77 (2004) arXiv:hep-th/0309167.

[85] D. Lüst, P. Mayr, R. Richter and S. Stieberger, Nucl. Phys. B 696, 205 (2004); A. Font and L. E. Ibanez, JHEP 0503, 040 (2005).

[86] Y. Kawamura, T. Kobayashi and T. Komatsu, Phys. Lett. B 400, 284 (1997) arXiv:hep$\mathrm{ph} / 9609462$.

[87] J. A. Maxin, V. E. Mayes and D. V. Nanopoulos, Phys. Rev. D 79, 066010 (2009) arXiv:0809.3200 [hep-ph]].

[88] J. A. Maxin, V. E. Mayes and D. V. Nanopoulos, Phys. Lett. B 690, 501 (2010) arXiv:0911.2806 [hep-ph]].

[89] J. R. Ellis, J. S. Hagelin, D. V. Nanopoulos and K. Tamvakis, Phys. Lett. B 125, 275 (1983).

[90] L. Alvarez-Gaume, J. Polchinski and M. B. Wise, Nucl. Phys. B 221, 495 (1983). 
[91] A. Djouadi, J. Kneur, and G. Moultaka, Comput. Phys. Commun. 176, (2007) 426-455. arXiv:hep-ph/0211331v2

[92] G. Belanger, F. Boudjema, A. Pukhov and A. Semenov, arXiv:1005.4133 [hep-ph].

[93] G. Belanger, F. Boudjema, A. Pukhov and A. Semenov, arXiv:0803.2360 [hep-ph].

[94] G. Belanger, F. Boudjema, A. Pukhov and A. Semenov, Comput. Phys. Commun. 176 (2007) 367 arXiv:hep-ph/0607059.

[95] [ATLAS and CDF and CMS and D0 Collaborations], arXiv:1403.4427 [hep-ex].

[96] P. A. R. Ade et al. [Planck Collaboration], Astron. Astrophys. 571, A16 (2014) arXiv:1303.5076 [astro-ph.CO]]. P. A. R. Ade et al. [Planck Collaboration], arXiv:1303.5076 [astro-ph.CO].

[97] G. Hinshaw et al. [WMAP Collaboration], Astrophys. J. Suppl. 208, 19 (2013) arXiv:1212.5226 [astro-ph.CO]].

[98] E. Barberio, et al (Heavy Flavor Averaging Group), arXiv:hep-ex/0704.3575v1

[99] M. Misiak et al, Phys. Rev. Lett. 98, 022002 (2007). arXiv:hep-ph/0609232v2

[100] RAaij et al. [LHCb Collaboration], Phys. Rev. Lett. 110, 021801 (2013) arXiv:1211.2674.

[101] G. W. Bennett et al (Muon g-2 Collaboration), Phys. Rev. Lett. 92, 161802 (2004). arXiv:hep-ex/0401008

[102] M. Davier, A. Hoecker, B. Malaescu and Z. Zhang, Eur. Phys. J. C 71, 1515 (2011) [Erratumibid. C 72, 1874 (2012)] arXiv:1010.4180 [hep-ph]].

[103] K. J. Bae, H. Baer and E. J. Chun, Phys. Rev. D 89, no. 3, 031701 (2014) arXiv:1309.0519 [hep-ph]].

[104] J. R. Ellis, J. L. Lopez and D. V. Nanopoulos, Phys. Lett. B 247, 257 (1990).

[105] E. W. Kolb, A. D. Linde and A. Riotto, Phys. Rev. Lett. 77 (1996) 4290 arXiv:hepph/9606260]; B. R. Greene, T. Prokopec and T. G. Roos, Phys. Rev. D 56 (1997) 6484 arXiv:hep-ph/9705357. E. W. Kolb, A. Riotto and I. I. Tkachev, Phys. Lett. B 423 (1998) 348 arXiv:hep-ph/9801306]; D. J. H. Chung, E. W. Kolb and A. Riotto, Phys. Rev. D 59 (1999) 023501 arXiv:hep-ph/9802238.

[106] H. Baer, V. Barger and P. Huang, JHEP 1111, 031 (2011) arXiv:1107.5581 [hep-ph]].

[107] E. Aprile et al. [XENON100 Collaboration], Phys. Rev. Lett. 107, 131302 (2011) arXiv:1104.2549 [astro-ph.CO]]. 
[108] E. Aprile et al. [XENON100 Collaboration], Phys. Rev. Lett. 109, 181301 (2012) arXiv:1207.5988 [astro-ph.CO]].

[109] M. Garny, A. Ibarra, M. Pato and S. Vogl, Phys. Rev. D 87, no. 5, 056002 (2013) arXiv:1211.4573 [hep-ph]].

[110] L. Baudis [DARWIN Consortium Collaboration], J. Phys. Conf. Ser. 375, 012028 (2012) arXiv:1201.2402 [astro-ph.IM]].

[111] M. Ackermann et al. [Fermi-LAT Collaboration], Phys. Rev. Lett. 107, 241302 (2011) arXiv:1108.3546 [astro-ph.HE]].

[112] A. Geringer-Sameth and S. M. Koushiappas, Phys. Rev. Lett. 107, 241303 (2011) arXiv:1108.2914 [astro-ph.CO]]. 